\title{
On strongly measure replete lattices and the general Wallman remainder
}

by

George Bachman (Brooklyn, N. Y.) and Mabel Szeto (Staten Island, N. Y.)

Abstract. Let $X$ be an abstract set and $\mathfrak{I}$ a lattice of subsets of $X$. $\mathfrak{L}$-tight regular measures are defined and their properties are investigated especially under mappings. Finally, tightness as well as $\sigma$-smoothness and $\tau$-smoothness are characterized in terms of the general Wallman remainder.

1. Introduction. Let $X$ be an abstract set and $\mathscr{L}$ a lattice of subsets of $X$. $M_{R}(\mathfrak{I})$ denotes the $\mathfrak{L}$-regular finitely additive measures on $\mathfrak{I}(\mathfrak{L})$, the algebra generated by $\mathfrak{L}$. In the first part of this paper, we define the set of $\mathfrak{L}$-tight measures $M_{R}^{t}(\mathfrak{L})$, and consider those lattices $\mathscr{L}$, for which $M_{R}^{\sigma}(\mathfrak{L})=M_{R}^{t}(\mathfrak{L})$, the strongly measure replete lattices. We consider how this property is preserved under lattice extension and lattice restriction, and then how it is preserved under "well-behaved" mappings between two sets. The general results extend in particular the work of Moran [12] on strongly measure compact spaces, and yield new results when applied to various specific lattices such as the closed sets in a topological space.

In the second part of the paper we see how the notion of an $\mathfrak{L}$-tight measure can be expressed in terms of induced measures on $I_{R}(\mathfrak{I})$, the general Wallman space associated with $X$. We investigate this relationship not only for $\mathfrak{L}$-tight measures but also for $\sigma$-smooth and $\tau$-smooth regular measures. For these general results we need only assume that $\mathscr{L}$ is a disjunctive lattice. This greatly extends the results of [4] where it was necessary to assume that $\mathfrak{L}$ was $\delta$ and normal in order to utilize the Alexandroff Representation Theorem [1]. Since there are many important topological lattices which are either not $\delta$ or not normal such as the closed sets in a $T_{1}$ topological space or the clopen sets in a $T_{2} 0$-dimensional space, these general results enable us to treat all these cases as well as the zero set lattice in a Tychonoff space and the Borel sets in a $T_{1}$ topological space as special settings for our general results.

We begin by defining the general notions involved and introducing the notations which will be used throughout. We also give a bit of background material in order to make the paper reasonably self-contained. 
2. Background and notation. We follow the notation and terminology in [1], [2], and [3]. Let $X$ be an abstract set and $\mathfrak{L}$ a lattice of subsets of $X$. It is assumed that $\varnothing, X \in \mathfrak{L}$, although this is not necessary for some of our results. We denote by:

1) $\mathfrak{A}(\mathfrak{L})$, the algebra generated by $\mathfrak{Q}$;

2) $\sigma(\mathfrak{I})$, the $\sigma$-algebra generated by $\mathfrak{L}$;

3) $\delta(\mathfrak{L})$, the lattice of all countable intersections of sets from $\mathfrak{L}$;

4) $\tau(\mathfrak{L})$, the lattice of arbitrary intersections of sets of $\mathfrak{L}$;

5) $\varrho(\mathfrak{L})$, the smallest class closed under countable intersections and unions which contains $\mathfrak{R}$;

6) $s(\mathfrak{L})$, the lattice derived Souslin sets.

Next, we denote by $M(\mathfrak{I})$ those finite valued finitely additive bounded measures on $\mathfrak{A}(\mathfrak{L})$. An element $\mu \in M(\mathfrak{I})$ is $\sigma$-smooth on $\mathfrak{L}$ if $L_{n} \in \mathfrak{L}, n=1,2, \ldots$, and $L_{n} \downarrow \varnothing$ implies $\mu\left(L_{n}\right) \rightarrow 0$. We say that $\mu$ is $\sigma$-smooth on $\mathfrak{A}(\mathfrak{L})$ (at times simply $\sigma$-smooth) if $A_{n} \in \mathfrak{A}(\mathfrak{S}), n=1,2, \ldots$, and $A_{n} \downarrow \varnothing$ implies $\mu\left(A_{n}\right) \rightarrow 0$. This is, of course, equivalent to saying that $\mu$ is countably additive.

We tacitly assume throughout that all measures are non-negative. This is, of course, no loss of generality since any $\mu \in M(\mathscr{L})$ can be split into its positive and negative pieces. We will also assume at times that any countably additive $\mu \in M(\mathfrak{L})$ has been extended uniquely to $\sigma(\mathfrak{L})$, and we denote the extension also by $\mu$.

Let $\mu \in M(\mathfrak{I}) ; \mu$ is $\mathfrak{R}$-regular if for any $A \in \mathfrak{A}(\mathfrak{I})$,

$$
\mu(A)=\sup \{\mu(L) \mid L \subset A, L \in \mathfrak{R}\} .
$$

It is easy to see that if $\mu$ is $\mathfrak{L}$-regular then $\mu$ is $\sigma$-smooth on $\mathfrak{U}(\mathfrak{E})$ if and only if it is $\sigma$-smooth on $\mathfrak{L}$. An element $\mu \in M(\mathfrak{L})$ is $\tau$-smooth on $\mathfrak{L}$ if for every net $\left\{L_{\alpha}\right\}, L_{\alpha} \in \mathfrak{R}$, such that $L_{\alpha} \downarrow \varnothing$, we have $\mu\left(L_{\alpha}\right) \rightarrow 0$.

We denote:

$M_{R}(\mathfrak{L})=$ the set of $\mathfrak{L}$-regular measures of $M(\mathfrak{I})$;

$M_{\sigma}(\mathfrak{L})=$ the set of $\sigma$-smooth measures on $\mathfrak{L}$ of $M(\mathfrak{L})$;

$M^{\sigma}(\mathfrak{L})=$ the set of $\sigma$-smooth measures on $\mathfrak{A}(\mathfrak{L})$ of $M(\mathfrak{L})$;

$M_{R}^{\sigma}(\mathfrak{L})=$ the set of $\mathfrak{L}$-regular measures of $M^{\sigma}(\mathfrak{L})$;

$M_{R}^{\mathfrak{\tau}}(\mathfrak{L})=$ the set of $\mathfrak{L}$-regular measures of $M(\mathfrak{L})$ which are also $\tau$-smooth on $\mathfrak{L}$.

$I(\mathfrak{L}), I_{R}(\mathfrak{L}), I_{R}^{\sigma}(\mathfrak{L})$, and $I_{R}^{\tau}(\mathfrak{L})$ are the subsets of the corresponding $M^{\prime}$ 's consisting of the non-trivial zero-one valued measures.

For $\mu \in M(\mathscr{L})$, the support of $\mu, S(\mu)=\bigcap\{L \in \mathbb{E} \mid \mu(L)=\mu(X)\}$.

$\mathfrak{L}$ is replete if for any $\mu \in I_{R}^{\sigma}(\mathfrak{Q}), \mu \neq 0, S(\mu) \neq \varnothing$.

We next recall some lattice terminology. $\mathfrak{L}$ is called:

a) complemented if $L \in \mathfrak{L}$ implies $L^{\prime} \in \mathfrak{L}$ (where prime denotes complement), that is, $\mathfrak{L}$ is an algebra.

b) separating if, for any two elements $x \neq y$ of $X$, there exists an element $L \in \mathfrak{L}$ such that $x \in L$ and $y \notin L$.

c) $T_{2}$ if, for any two elements $x \neq y$ of $X$, there exist $A, B \in \mathfrak{L}$ such that $x \in A^{\prime}$ and $y \in B^{\prime}$ and $A^{\prime} \cap B^{\prime}=\varnothing$. d) disjunctive if for any $x \in X$ and $A \in \mathfrak{L}$ such that $x \notin A$, there exists a $B \in \mathfrak{L}$ such that $x \in B$ and $A \cap B=\varnothing$.

e) regular if for any $x \in X$, and $A \in \mathfrak{L}$ such that $x \notin A$ there exist $B, C \in \mathfrak{L}$ such that $x \in B^{\prime}, A \subset C^{\prime}$ and $B^{\prime} \cap C^{\prime}=\varnothing$.

f) normal, if for any $A, B \in \mathfrak{L}$ such that $A \cap B=\varnothing$ there exist $C, D \in \mathfrak{Q}$ with $A \subset C^{\prime}, B \subset D^{\prime}$, and $C^{\prime} \cap D^{\prime}=\varnothing$.

g) delta lattice $(\delta$-lattice) if $\delta(\mathfrak{S})=\mathfrak{L}$.

h) compact if for any collection $\left\{L_{\alpha}\right\}$ of sets of $\mathfrak{L}, \cap L_{\alpha}=\varnothing$ implies there exists a finite subcollection with empty intersection.

Similarly we define $\mathfrak{I}$ countably compact or Lindelöf.

i) countably paracompact if for every sequence $\left\{A_{n}\right\}$ of sets of $\mathfrak{L}$ such that $A_{n} \downarrow \varnothing$, there exists a sequence $\left\{B_{n}\right\}$ of sets of $\mathfrak{L}$ such that, for all $n, A_{n} \subset B_{n}^{\prime}$ and $B_{n}^{\prime} \downarrow \varnothing$.

Let $\mathfrak{S}_{1}$ and $\mathfrak{\Omega}_{2}$ be two lattices of subsets of $X$. $\mathfrak{L}_{1}$ semi-separates $\mathfrak{L}_{2}$ if $A \in \mathfrak{I}_{1}, B \in \mathfrak{I}_{2}$ and $A \cap B=\emptyset$ implies there exists $C \in \mathfrak{I}_{1}, B \subset C$, and $A \cap C$ $=\varnothing . \mathfrak{I}_{1}$ separates $\mathfrak{I}_{2}$ if $A, B \in \mathfrak{I}_{2}$ and $A \cap B=\varnothing$ implies there exist $C, D \in \mathfrak{I}_{1}$ such that $A \subset C, B \subset D$ and $C \cap D=\varnothing$. $\mathfrak{\Omega}_{2}$ is $\mathfrak{\Omega}_{1}$-countably paracompact $\left(\mathfrak{\Omega}_{1}-c b\right)$ if for any sequence $\left\{B_{n}\right\}$ of sets of $\mathfrak{L}_{2}$ with $B_{n} \downarrow \varnothing$, there exists a sequence $\left\{A_{n}\right\}$ of sets of $\mathfrak{L}_{1}$ with $B_{n} \subset A_{n}^{\prime}\left(B_{n} \subset A_{n}\right)$ and $A_{n}^{\prime} \downarrow \varnothing\left(A_{n} \downarrow \varnothing\right)$.

If $K$ is a subset of $X, K$ is called $\mathcal{Q}$-compact if the lattice

$$
K \cap \mathfrak{L}=\{K \cap A: A \in \mathfrak{L}\}
$$

is compact. Similarly, we define $K$ to be $\mathfrak{Q}$-countably compact, etc.

$C(\mathfrak{L})$ will designate the set of all real-valued $\mathfrak{L}$-continuous functions defined on $X$, where $f: X \rightarrow R$ is called $\mathfrak{L}$-continuous if $f^{-1}(E) \in \mathfrak{L}$ for any closed set $E \subset R .3(\mathbb{R})$ designates the lattice of zero sets of functions in $C(\mathbb{I})$.

If $X$ is a topological space, $\mathfrak{J}_{X}$ designates the lattice of closed sets. We alsc write $3_{X}=3\left(\mathfrak{J}_{X}\right)$ in this case. Also, $\mathfrak{\Omega}_{X}$ designates the compact subset of $X$.

Now let $X$ be an abstract set and $\mathfrak{L}$ a lattice of subsets. If $x \in X$, then $\mu_{x}$ is the measure concentrated at $x$ so $\mu_{x}(A)=\left\{\begin{array}{ll}1 & \text { if } x \in A \\ 0 & \text { if } x \notin A\end{array}\right.$ where $A \in \mathfrak{Q}(\mathfrak{I}) . \mu_{x} \in I_{R}(\mathfrak{L})$ if and only if $\mathfrak{L}$ is disjunctive. This gives rise to a mapping $\mathfrak{E}: X \rightarrow I_{R}(\mathfrak{L})$ where $\mathfrak{E}(x)=\mu_{x}$, for $x \in X$, if $\mathfrak{L}$ is disjunctive. $\mathfrak{E}$ will be one-to-one if $\mathfrak{I}$ is separating. If $\mathfrak{L}$ is separating and disjunctive and if $X$ is given the $\tau(\mathfrak{L})$ topology and $I_{R}(\mathfrak{L})$ is given the Wallman topology, then $\mathbb{E}$ is a homeomorphism of $X$ into $I_{R}(\mathfrak{L})$ (see [2] for details). The Wallman topology is obtained by taking the totality of all $W(L)=\left\{\mu \in I_{R}(\mathfrak{L}) \mid \mu(L)=1\right\}$ where $L \in \mathbb{L}$ as a base for the closed sets. For a disjunctive $\mathscr{L}, I_{R}(\mathscr{L})$ is always a compact $T_{1}$ space and will be $T_{2}$ if and only if $\mathfrak{L}$ is normal, and is called the general Wallman space associated with $X$ and $\mathfrak{L}$. If $X$ is a topological space and $\mathfrak{L}$ a particularly chosen lattice, $I_{R}(\mathfrak{L})$ clearly yields well-known compactifications of $X$.

If $\mathfrak{L}$ is separating and disjunctive, we will, in the sequel, identify $X$ with its image $\mathfrak{E}(X)$ in $I_{R}(\mathscr{L})$. Also, in this case, it is easy to see that $I_{R}^{\mathfrak{\tau}}(\mathscr{L})=X$. We also 
make use of the fact that if $\mu \in M_{R}^{\sigma}(\mathfrak{I})$, then its extension to $\sigma(\mathfrak{L})$ is $\delta(\mathfrak{L})$-regular, and, consequently, $\mathfrak{L}$-regular if $\mathfrak{L}$ is a delta lattice; in fact, if $\mathfrak{L}$ is a delta lattice, $\mu^{*}$ is $\mathfrak{L}^{\prime}$ outer-regular on all subsets and therefore $\mathfrak{L}$-regular on the $\mu^{*}$-measurable sets, in particular on $\sigma(\mathfrak{I})$.

Definition $1 . X$ is $\mathfrak{L}$-measure replete (or simply $\mathfrak{L}$ is measure replete) if for any $\mu \in M_{R}^{\sigma}(\mathscr{L}), \mu \neq 0, S(\mu) \neq \varnothing$.

THEOREM 2.1. If $\mathfrak{L}$ is a $\delta$-lattice and if $\mu \in M_{R}^{\sigma}(\mathfrak{L})$, then $\mu \in M_{R}^{\tau}(\mathfrak{L})$ if and only if for any net $\left\{L_{\alpha}\right\}$ of $\mathfrak{I}$ with $L_{\alpha} \downarrow, \mu^{*}\left(\cap L_{\alpha}\right)=\inf \mu\left(L_{\alpha}\right)$. Also, $\mu \in M_{R}^{\tau}(\mathbb{I})$ if and only if for any net $\left\{L_{\alpha}\right\}$ of $\mathfrak{I}$ which is a filter base, $\mu^{*}\left(\cap L_{\alpha}\right)=\inf \mu\left(L_{\alpha}\right)$.

Proof. See [15].

THEOREM 2.2. If $\mathfrak{L}$ is a $\delta$-lattice, then $M_{R}^{\sigma}(\mathfrak{I})=M_{R}^{\mathfrak{\tau}}(\mathfrak{I})$ if and only if $S(\mu) \neq \varnothing$ for all $\mu \in M_{R}^{\sigma}(\mathbb{\Omega}), \mu>0$.

Proof. Let $\mu \in M_{R}^{\mathfrak{\tau}}(\mathfrak{L})$. Since $\{L \in \mathfrak{L} \mid \mu(L)=\mu(X)\}$ is a filter base with intersection $S(\mu)$, we have $\mu^{*}(S(\mu))=\mu(X)$ by Theorem 2.1 .

Conversely, let $\mu \in M_{R}^{\sigma}(\mathfrak{L}), \mu>0$. Suppose $\mu \notin M_{R}^{\tau}(\mathfrak{L})$. Then there exists a net $H=\left\{L_{\alpha}^{\prime}\right\} \uparrow X, L_{\alpha} \in \mathbb{R}$, such that $\sup _{\alpha} \mu\left(L_{\alpha}^{\prime}\right)=a<\mu(X)$. There exists a subsequence $\left\{L_{\alpha_{n}}^{\prime}\right\} \uparrow$ such that $\lim \mu\left(L_{\alpha_{n}}^{\prime}\right)=a<\mu(X)$. Let $\tilde{L}=\bigcap L_{\alpha_{n}} \in \mathfrak{L}$ since $\mathfrak{L}$ is a $\delta$-lattice. Define $\varrho$ on $\sigma(\mathfrak{I})$ by $\varrho(B)=\mu(B \cap \tilde{L})$ where $B \in \sigma(\mathfrak{L})$. It can be shown that $\varrho \in M_{R}^{\sigma}(\mathscr{L})$ and that $S(\varrho) \subset \tilde{L}$. Also, $S(\varrho) \neq \varnothing$ by hypothesis. Let $x \in S(\varrho)$ so $x \in \tilde{L}$, and $x \in L_{\beta}^{\prime}$ where $L_{\beta}^{\prime}$ is in the original family $H$ and $\beta \neq \alpha_{n}$ for any $n$. If $\varrho\left(L_{\beta}^{\prime}\right)=0$, then $\varrho\left(L_{\beta}\right)=\varrho(X)$. Hence, $x \in S(\varrho) \subset L_{\beta}$ a contradiction. Therefore, we must have that $\varrho\left(L_{\beta}^{\prime}\right)>0$. Then

$$
\mu\left(L_{\beta}^{\prime} \cup \bigcup L_{\alpha_{n}}^{\prime}\right)=\mu\left(L_{\beta}^{\prime} \cup \tilde{L}^{\prime}\right)=\mu\left(\tilde{L}^{\prime}\right)+\mu\left(L_{\beta}^{\prime} \cap \tilde{L}\right)=\mu\left(\bigcup L_{\alpha_{n}}^{\prime}\right)+\varrho\left(L_{\beta}^{\prime}\right)>a,
$$

which is a contradiction since $L_{\beta}^{\prime} \cup L_{\alpha_{n}}^{\prime} \subset L_{\beta_{n}}^{\prime}$ where $\beta, \alpha_{n}<\beta_{n}$ and $L_{\beta_{n}}^{\prime}$ is in $H$, and $\sup \mu\left(L_{\alpha}^{\prime}\right)=a$. Thus $\mu \in M_{R}^{\tau}(\mathfrak{I})$.

Now we can apply the above results to the following cases:

(1) Let $\mathfrak{L}=3_{X}$ and $X$ be a $T_{3 \frac{1}{2}}$ space. Then (A): $\mu \in M_{R}^{\tau}(\mathfrak{R})$ iff for any $Z_{\alpha} \in 3_{X}, Z_{\alpha} \downarrow, \mu^{*}\left(\cap Z_{\alpha}\right)=\inf \mu\left(Z_{\alpha}\right)$. (B): $\mu \in M_{R}^{\tau}(\mathbb{I})$ iff for $Z_{\alpha} \in \mathcal{B}_{X},\left\{Z_{\alpha}\right\}$ is a filter base, $\mu^{*}\left(\cap Z_{\alpha}\right)=\inf \mu\left(Z_{\alpha}\right)$. This generalizes a result of Varadarajan [16].

(2) Let $X$ be a topological space and $\mathfrak{I}=\mathfrak{J}_{X}$. Then (A): $\mu \in M_{R}^{\tau}(\mathfrak{I})$ iff for $F_{\alpha} \in \mathfrak{J}_{X}, F_{\alpha} \downarrow, \mu^{*}\left(\cap F_{\alpha}\right)=\inf \mu\left(F_{\alpha}\right)$. (B): $\mu \in M_{R}^{\tau}(\mathfrak{I})$ iff for $F_{\alpha} \in \mathfrak{J}_{X},\left\{F_{\alpha}\right\}$ is a filter base, $\mu^{*}\left(\cap F_{\alpha}\right)=\inf \mu\left(F_{\alpha}\right)$. This generalizes a result of Gardner [8].

(3) Let $\mathfrak{L}=\mathfrak{B}_{X}$ and $X$ be a $T_{3 \frac{1}{2}}$ space. Then $X$ is measure compact, (i.e., $\left.M_{R}^{\sigma}(3)=M_{R}^{\tau}(3)\right)$ iff $S(\mu) \neq \varnothing$ for any $\mu \in M_{R}^{\sigma}(3), \mu>0$. This yields as a special case a theorem of Moran [13].

(4) Let $X$ be a topological space and $\mathfrak{L}=\mathfrak{J}_{X}$. Then $\mathfrak{I}_{X}$ is Borel measure compact (i.e., $\left.M_{R}^{\sigma}(\mathfrak{I})=M_{R}^{\tau}(\mathfrak{I})\right)$ iff $S(\mu) \neq \varnothing$ for any $\mu \in M_{R}^{\sigma}(\mathfrak{I}), \mu>0$. This yields a special case of Gardner [8].

Defintion 2. Let $\mu \in M_{R}^{\sigma}(\mathfrak{L}), \mu \geqslant 0$. Then $\mu$ is called $\mathfrak{L}$-tight if for every $\varepsilon>0$, there exists $K \in \mathfrak{R}=\mathfrak{L}$-compact sets such that $\mu_{*}\left(K^{\prime}\right) \leqslant \varepsilon$. The collection of $\mathfrak{L}$-tight measures is denoted by $M_{R}^{t}(\mathfrak{L})$.
Note. $\mu_{*}\left(K^{\prime}\right) \leqslant \varepsilon \Leftrightarrow \mu^{*}(K) \geqslant \mu(X)-\varepsilon$.

DefintTION 3. $X$ is $\mathfrak{R}$-strongly measure replete (simply $\mathfrak{L}$ is strongly measure replete) if $M_{R}^{\sigma}(\mathfrak{L})=M_{R}^{t}(\mathfrak{L})$.

THEOREM 2.3. $M_{R}^{\mathrm{t}}(\mathfrak{L}) \subset M_{R}^{\tau}(\mathfrak{L}) \subset M_{R}^{\sigma}(\mathfrak{I}) \subset M_{R}(\mathfrak{L})$.

Proof. The proof is not difficult and will be omitted.

THeorem 2.4. Let $\mathfrak{Q}$ be a $\delta$-lattice, and let $\mu \in M_{R}^{\mathfrak{\tau}}(\mathfrak{S})$. Then for any $\nu \in M_{R}(\tau \mathfrak{L})$ extending $\mu, \nu \leqslant \mu^{*}$ on $\tau \mathscr{L}$. If $\nu \in M_{R}^{\tau}(\tau \mathscr{L})$, then $\nu=\mu^{*}$ on $\tau \mathscr{Q}$.

Proof. Let $F=\bigcap L_{\alpha} \in \tau \mathfrak{Q}, L_{\alpha} \in \mathfrak{L}$ and $L_{\alpha} \downarrow$. Then $v(F) \leqslant v\left(L_{\alpha}\right)=\mu\left(L_{\alpha}\right)$. Since $\mathfrak{L}$ is a $\delta$-lattice, we have $v(F) \leqslant \inf \mu\left(L_{\alpha}\right)=\mu^{*}\left(\cap L_{\alpha}\right)=\mu^{*}(F)$. Hence, $v \leqslant \mu^{*}$ on $\tau \mathscr{L}$. If $\nu \in M_{R}^{\tau}(\tau \mathscr{L})$, then $\nu(F)=\inf v\left(L_{\alpha}\right)=\inf \mu\left(L_{\alpha}\right)=\mu^{*}(F)$ so $v=\mu^{*}$ on $\tau \mathfrak{L}$.

Note. (1) If $\mathfrak{L}$ is separating and disjunctive, and if $\mu \in M_{R}^{\tau}(\mathfrak{L})$, then we will show that $\mu$ extends uniquely to a $\nu \in M_{R}^{\tau}(\tau \mathfrak{L}$ ) (see Theorem 5.7).

(2) It is easy to see that if $\mathfrak{L}$ is separating, disjunctive and normal (or if $\mathfrak{I}$ is ust $T_{2}$ ), then $\mathbb{R}=$ collection of $\mathfrak{L}$-compact sets is contained in $\tau \mathscr{L}$.

THEOREM 2.5. If $\mathfrak{L}$ is separating, disjunctive and normal (or if $\mathfrak{I}$ is separating, disjunctive and $\left.T_{2}\right)$ and if $\mu \in M_{R}^{t}(\mathcal{Q})$, then there exists a unique extension of $\mu$ to $\nu \in M_{\mathbb{R}}^{\tau}(\tau \mathfrak{I})$ and even $v \in M_{\mathbb{R}}^{t}(\tau \mathfrak{I})$. Also, every $A \in \sigma(\tau \mathfrak{I})$ is $\mathfrak{R}$-regular with respect to $v$, where $\mathfrak{R}=$ the collection of $\mathfrak{\Omega}$-compact sets.

Proof. Since $\mu \in M_{R}^{t}(\mathfrak{L}), \mu \in M_{R}^{\tau}(\mathfrak{L})$ by Theorem 2.3 , and by note (1) above, $\mu$ extends uniquely to $v \in M_{R}^{\tau}(\tau \mathfrak{I})$. Also by note $(2), \mathfrak{K} \subset \tau \mathfrak{L}$. Let $A \in \sigma(\tau \mathfrak{L})$. Then there exists $F \subset A, F \in \tau \mathfrak{L}$ such that $v(A-F)<\frac{1}{2} \varepsilon$ where $\varepsilon>0$. Since $\mu$ is $\mathfrak{L}$-tight, there exists $K \in \mathfrak{R} \subset \tau \mathfrak{L}$ such that $\mu^{*}(K) \geqslant \mu(X)-\frac{1}{2} \varepsilon$. By Theorem 2.4, $v=\mu^{*}$ on $\tau \mathscr{\Omega}$, so $\nu(K) \geqslant \mu(X)-\frac{1}{2} \varepsilon$. Therefore $\nu(X-K) \leqslant \frac{1}{2} \varepsilon$. Now consider $\widetilde{K}=K \cap F$ $\in \tau \mathfrak{L}, \widetilde{K} \in \mathfrak{R}$, and $\tilde{K} \subset F \subset A$ and, since $F-\tilde{K}=F-K$,

$$
v(A-\tilde{K}) \leqslant v(A-F)+v(F-\tilde{K}) \leqslant v(A-F)+v(X-K) \leqslant \frac{1}{2} \varepsilon+\frac{1}{2} \varepsilon=\varepsilon,
$$

which completes the proof

Combining Theorem 2.5 and Theorem 2.3 in [3], we have:

THEOREM 2.6. If $\mathfrak{R}$ is $\delta$, separating, disjunctive and normal (or if $\mathfrak{L}$ is $\delta$, separating, disjunctive and $T_{2}$ ) and if $\sigma(\mathfrak{L}) \subset s(\mathfrak{I})$, then for $\mu \in M^{\sigma}(\mathfrak{L})$, we have (1) $\mu \in M_{\mathbb{R}}^{\sigma}(\mathfrak{L})$, (2) if $\mu$ is also $\mathfrak{\Omega}$-tight, then there exists a unique extension of $\mu$ to $v \in M_{\mathbb{R}}^{\tau}(\tau \mathbb{S})$ and even $\nu \in M_{R}^{t}(\tau \mathscr{\Omega})$, and every $A \in \sigma(\tau \mathfrak{I})$ is $\Omega$-regular with respect to $v$.

COROLlaRY. If $X$ is a metric space, then (1) every Borel measure is $\mathfrak{I}_{X}$-regular; (2) if $\mu$ is a tight Borel measure, then every Borel set is $\boldsymbol{\Omega}_{\mathbf{X}}$-regular with respect to $\mu$. Proof. The conditions of Theorem 2.6 are already satisfied here for $\mathfrak{L}$ $=\tau \mathfrak{L}=\mathfrak{I}_{X}$.

3. Extension and restriction. In this section we investigate the behavior of strongly measure replete when the lattice is enlarged or restricted.

2- Fundamenta Mathematicae $\mathrm{CXXII/3}$ 
Throrem 3.1 (General Extension Theorem). Let $\mathfrak{I}_{1} \subset \mathfrak{\Omega}_{2}$ be lattices of subsets of $X$. Then any $\mu \in M_{R}\left(\mathfrak{I}_{1}\right)$ can be extended to a $v \in M_{R}\left(\mathfrak{L}_{2}\right)$. If $\mu \in M_{R}^{\sigma}\left(\mathfrak{I}_{1}\right)$, then $v \in M_{R}^{\sigma}\left(\mathfrak{\Omega}_{2}\right)$ if $\mathfrak{\Omega}_{2}$ is $\mathfrak{\Omega}_{1}$ countably paracompact. This will also be true if $\mathfrak{\Omega}_{1}$ is a $\delta$-lattice and $\mathfrak{S}_{2}$ is just $\sigma\left(\mathfrak{I}_{1}\right)$ countably paracompact, or even just $S_{\mu}^{*}$. colintably paracompact where $S_{\mu}^{*}$ are the $\mu^{*}$-measurable sets.

\section{Proof. See [5].}

THEOREM 3.2. Let $\mathfrak{\Omega}_{1}$ and $\Omega_{2}$ be. $\delta$-lattices of subsets of an abstract set $X$ such that $\mathfrak{I}_{1} \subset \mathfrak{I}_{2} \subset \tau \mathfrak{I}_{1}$. Then if (a) $\sigma\left(\mathfrak{I}_{1}\right) \subset s\left(\mathfrak{S}_{1}\right)$, or if (b) $\mathfrak{I}_{1}$ semi-separates $\mathfrak{I}_{2}$ then $\mathfrak{I}_{1}$ strongly measure replete implies that $\mathfrak{S}_{2}$ is strongly measure replete.

Proof. In either case, if $v \in M_{R}^{\sigma}\left(\Omega_{2}\right)$ then its restriction $\mu=\left.v\right|_{\mathscr{Q}\left(\Omega_{1}\right)} \in M_{R}^{\sigma}\left(\Omega_{1}\right)$ (see [3]). Since $\mathfrak{L}_{1}$ is strongly measure replete, there exists $K \in \mathfrak{N}=\mathfrak{L}_{1}$-compact sets such that

(1)

$$
\mu^{*}(K) \geqslant \mu(X)-\varepsilon=\nu(X)-\varepsilon .
$$

Now $K$ being $\mathfrak{I}_{1}$-compact implies that $K$ is $\tau \mathfrak{Q}_{1}$-compact, and therefore $K$ is $\mathfrak{I}_{2}$-compact. Since $\mathfrak{I}_{1}$ and $\mathfrak{I}_{2}$ are $\delta$-lattices, if $E \subset X$ then

and

$$
\begin{aligned}
\mu^{*}(E) & =\inf \mu\left(L_{1}^{\prime}\right) & & \text { where } E \subset L_{1}^{\prime}, L_{1} \in \mathbb{\Omega}_{1} \\
& =\inf v . & & \text { where } E \subset L_{1}^{\prime}, L_{1} \in \mathbb{\Omega}_{1}
\end{aligned}
$$$$
v^{*}(E)=\inf v\left(L_{2}^{\prime}\right) \quad \text { where } E \subset L_{2}^{\prime}, L_{2} \in \mathfrak{I}_{2}
$$

Therefore $v^{*}(E) \leqslant \mu^{*}(E)$, and in particular, $v^{*}(K) \leqslant \mu^{*}(K)$. But if $K \subset L_{2}^{\prime}, L_{2} \in \Omega_{2}$, then since $L_{2}=\bigcap_{\alpha} L_{1, \alpha}, L_{1, \alpha} \in \mathfrak{I}_{1}$, we have $K \subset \bigcup_{\alpha} L_{1, \alpha}^{\prime}$, and since $K$ is $\mathfrak{L}_{1}$-compact, $K \subset \bigcup_{1}^{n} L_{1, \alpha_{i}}^{\prime}=\hat{L}_{1}^{\prime}, \hat{L}_{1} \in \mathfrak{\Omega}_{1}$ and $\hat{L}_{1}^{\prime} \subset L_{2}^{\prime}$. Hence, $\nu^{*}(K)=\mu^{*}(K)$ and (1) implies that $v^{*}(K) \geqslant v(X)-\varepsilon$ and, since $K$ is $\mathfrak{\Omega}_{2}$-compact, it follows that $\mathfrak{Q}_{2}$ is strongly measure replete:

THEOREM 3.3. Let $\mathfrak{S}_{1}$ and $\mathfrak{I}_{2}$ be $\delta$-lattices of subsets of an abstract set $X$ such that $\mathfrak{I}_{1} \subset \mathfrak{Q}_{2}$. If (a) $\mathfrak{I}_{2}$ is $\mathfrak{\Omega}_{1}$ countably paracompact or, more generally, if $\mathfrak{\Omega}_{2}$ is just $\sigma\left(\mathfrak{S}_{1}\right)$ countably paracompact, or if (b) $\mathfrak{\Omega}_{2}$ is countably paracompact and $\mathfrak{\Omega}_{1}$ separates $\mathfrak{L}_{2}$, then $\mathfrak{L}_{2}$ strongly measure replete implies that $\mathfrak{I}_{1}$ is strongly measure replete.

Proof. In case (b) it is easy to see that $\mathfrak{L}_{2}$ is $\mathfrak{\Omega}_{1}$ countably paracompact so we need just consider case (a). Let $\mu \in M_{R}^{\sigma}\left(\mathfrak{L}_{1}\right)$. By Theorem 3.1, we can extend $\mu$ to $v \in M_{R}^{\sigma}\left(\mathfrak{L}_{2}\right)$. Since $\mathfrak{\Omega}_{2}$ is strongly measure replete, for any $\varepsilon>0$, there exists $K$, $\mathfrak{S}_{2}$-compact, such that $\nu^{*}(K) \geqslant \nu(X)-\varepsilon=\mu(X)-\varepsilon$. But $K$ is $\mathfrak{S}_{1}$-compact and as before (see proof of Theorem 3.2) $\mu^{*}(K) \geqslant \nu^{*}(K)$. Thus $\mu^{*}(K) \geqslant \mu(X)-\varepsilon$, and $\mathfrak{L}_{1}$ is strongly measure replete.

Now we have the following applications:

(1) Let $X$ be a $T_{3+1}$ space. Let $\mathfrak{S}_{1}=3_{X}$ and $\mathfrak{S}_{2}=\mathfrak{I}_{x}$. Then by Theorem 3.2, part (a), $X$ strongly measure compact (i.e., $3_{X}$ strongly measure replete) $\Rightarrow \mathfrak{I}_{X}$ strongly measure replete.
(2) If $X$ is countably paracompact and normal, then $\mathfrak{I}_{X}^{\prime}$ strongly measure replete $\Rightarrow 3 X$ is strongly measure replete (i.e., $X$ strongly measure compact) by Theorem 3.3, part (b).

4. Mappings. In this section we will present a number of mapping theorems between spaces of regular lattice measures and then we will show how these results can be applied to questions on the preservation of strong measure repleteness. Let $\mathfrak{L}_{1}$ be a lattice of subsets of $X$ and $\Omega_{2}$ a lattice of subsets of $Y$. A mapping $T: X \rightarrow Y$ is $\mathfrak{I}_{1}-\mathfrak{\Omega}_{2}$ continuous if $T^{-1}\left(\mathfrak{\Omega}_{2}\right)$ is contained in $\mathfrak{\Omega}_{1}$. It is $\mathfrak{\Omega}_{1}-\mathfrak{L}_{2}$ closed if $T\left(\mathfrak{\Omega}_{1}\right) \subset \mathfrak{I}_{2}$. If $T$ is a surjection which is $\mathfrak{I}_{1}-\mathfrak{I}_{2}$ continuous and closed such that $T^{-1}\{y\}$ is $\mathfrak{I}_{1}$-compact for any $y \in Y$, then $T$ is called $\mathfrak{I}_{1}-\mathfrak{I}_{2}$ perfect.

THEOREM 4.1. Let $\mathfrak{I}_{1}$ be a $\delta$-lattice of subsets of $X$ and $\mathfrak{I}_{2}$ a $\delta$-lattice of subsets of $Y$. Suppose $T: X \rightarrow Y$ is $\mathfrak{\Omega}_{1}-\mathfrak{\Omega}_{2}$ continuous and that $\hat{T}: M_{R}^{\sigma}\left(\mathfrak{I}_{1}\right) \rightarrow M_{R}^{\sigma}\left(\mathfrak{\Omega}_{2}\right)$ where $\hat{T}$ is defined for $\mu \in M_{R}^{\sigma}\left(\mathfrak{\Omega}_{1}\right)$, by $\hat{T} \mu=\mu T^{-1}$, then $\hat{T}: M_{R}^{t}\left(\mathfrak{L}_{3}\right) \rightarrow M_{R}^{t}\left(\mathfrak{L}_{2}\right)$.

Proof. Let $\mu \in M_{R}^{t}\left(\mathfrak{Q}_{1}\right)$. Then there exists an $\mathfrak{\Omega}_{1}$-compact set, $K_{1}$, such that $\mu^{*}\left(K_{1}\right) \geqslant \mu(X)-\varepsilon$ where $\varepsilon>0$. Clearly $T\left(K_{1}\right)$ is $\mathbb{S}_{2}$-compact. Let $v=\widehat{T} \mu=\mu T^{-1}$. Then $v^{*}\left(T K_{1}\right)=\inf \mu T^{-1}\left(L_{2}^{\prime}\right)$ where $T K_{1} \subset L_{2}^{\prime}, L_{2} \in \mathfrak{L}_{2}$. But $T K_{1} \subset L_{2}^{\prime}$ if and only if $K_{1} \subset T^{-1} T K_{1} \subset T^{-1}\left(L_{2}\right)^{\prime}=T^{-1}\left(L_{2}^{\prime}\right)$. However,

$$
\begin{aligned}
\mu^{*}\left(K_{1}\right) & =\inf \mu\left(L_{1}^{\prime}\right) & & \text { where } K_{1} \subset L_{1}^{\prime}, L_{1} \in \mathfrak{I}_{1} \\
& \leqslant \inf \mu T^{-1}\left(L_{2}^{\prime}\right) & & \text { where } K_{1} \subset T^{-1}\left(L_{2}^{\prime}\right), L_{2} \in \mathfrak{I}_{2} .
\end{aligned}
$$

Hence, $\mu^{*}\left(K_{1}\right) \leqslant v^{*}\left(T K_{1}\right)$. Also, $v(Y)=\mu T^{-1} Y=\mu(X)$. Therefore, $v^{*}\left(T K_{1}\right)$ $\geqslant \mu^{*}\left(K_{1}\right) \geqslant \mu(X)-\varepsilon=\nu(Y)-\varepsilon$, so $v=\widehat{T} \mu \in M_{R}^{t}\left(\mathfrak{I}_{2}\right)$.

Note. The condition $\hat{T}: M_{R}^{\sigma}\left(\mathfrak{I}_{1}\right) \rightarrow M_{R}^{\sigma}\left(\mathfrak{\Omega}_{2}\right)$ will be satisfied if $\mathfrak{L}_{2}$ is a $\delta$-lattice and $\sigma\left(\mathfrak{L}_{2}\right) \subset s\left(\mathfrak{L}_{2}\right)$. It will also be satisfied if $T^{-1}\left(\mathfrak{L}_{2}\right)$ semi-separates $\mathfrak{I}_{1}$, e.g., if $T$ is also $\mathfrak{Q}_{1}-\mathfrak{Q}_{2}$ closed.

THEOREM 4.2. (1) If $T: X \rightarrow Y$ is $\mathfrak{S}_{1}-\mathfrak{Q}_{2}$ continuous and surjective and if $v \in M_{R}\left(\mathfrak{I}_{2}\right)$, there exists $\varrho \in M_{R}\left(\Omega_{1}\right)$ such that $v=\varrho T^{-1}=\hat{T}_{\varrho}$. (2) If in addition, $v \in M_{R}^{\sigma}\left(\mathfrak{\Omega}_{2}\right)$ and if $\mathfrak{\Omega}_{1}$ is $T^{-1}\left(\mathfrak{\Omega}_{2}\right)$ countably paracompact or if $\mathfrak{\Omega}_{1}$ is $T^{-1}\left(\mathfrak{\Omega}_{2}\right) c b$, then there exists $\varrho \in M_{R}^{\sigma}\left(\mathfrak{L}_{1}\right)$ such that $v=\varrho T^{-1}=\hat{T} \varrho$.

Proof. For $\nu \in M_{R}\left(\mathfrak{L}_{2}\right)$ we define $\mu T^{-1}(A)=v(A)$, where $A \in \mathfrak{Q}\left(\mathfrak{L}_{2}\right)$. This defines $\mu$ on $T^{-1}\left(\mathfrak{A}\left(\mathfrak{I}_{2}\right)\right)=\mathfrak{A}\left(T^{-1}\left(\mathfrak{\Omega}_{2}\right)\right)$ and $\mu$ is well-defined for if $T^{-1}(A)$ $=T^{-1}(B)$, then since $T$ is surjective, $A=B$. Also $\mu \in M_{R}\left(T^{-1}\left(\mathfrak{I}_{2}\right)\right)$ and by Theorem 3.1, $\mu$ can be extended to $\varrho \in M_{R}\left(\mathfrak{Q}_{1}\right)$. Now for $A \in \mathfrak{U}\left(\mathfrak{I}_{2}\right), \varrho T^{-1}(A)=\mu T^{-1}(A)$ $=\nu(A)$. Hence $v=\varrho T^{-1}=\hat{T} \varrho$. For part (2) the proof is straightforward.

THEOREM 4.3. Let $\mathfrak{I}_{1} \subset \mathfrak{\Omega}_{3} \subset \tau \mathfrak{\Omega}_{1}$ be lattices of subsets of $X$.where $\mathfrak{I}_{\mathbf{1}}$ and $\mathfrak{L}_{3}$ are $\delta$-lattices and $\sigma\left(\mathfrak{\Omega}_{1}\right) \subset s\left(\mathfrak{\Omega}_{1}\right)$ or $\mathfrak{\Omega}_{1}$ semi-separates $\mathfrak{\Omega}_{3}$. Let $\mathfrak{\Omega}_{2} \subset \mathfrak{\Omega}_{4} \subset \tau \mathfrak{\Omega}_{2}$ be lattices of subsets of $Y$ such that $\mathfrak{\Omega}_{4}$ is $\mathfrak{\Omega}_{2}$ countably paracompact (or cb) and where $\mathfrak{I}_{2}$ and $\mathfrak{I}_{4}$ are $\delta$-lattices. Let (a) $T: X \rightarrow Y$ be $\mathfrak{S}_{3}-\mathfrak{\Omega}_{4}$ continuous and surjective, and let $\Omega_{3}$ be $T^{-1}\left(\mathfrak{\Omega}_{4}\right)$ countably paracompact (or $c b$ ), then $\mathfrak{I}_{1}$ strongly measure replete implies $\mathfrak{S}_{2}$ strongly measure replete. In particular, if (b) $T$ is $\mathfrak{S}_{3}-\mathfrak{I}_{4}$ perfect, then $\mathfrak{L}_{1}$ strongly measure replete implies $\mathfrak{S}_{2}$ strongly measure replete. 
Proof. Since $\mathfrak{I}_{4}$ is $\mathfrak{L}_{2}$ countably paracompact, $\mathfrak{S}_{4}$ is countably paracompact, and in case (b) it can be shown that $\mathfrak{\Omega}_{3}$ is $T^{-1}\left(\mathfrak{I}_{4}\right)$ countably paracompact (see [3]), so we need just consider case (a). Let $v \in M_{R}^{\sigma}\left(\mathfrak{\Omega}_{2}\right)$; then by Theorem 3.1, $v$ can be extended to $\varrho \in M_{R}^{\sigma}\left(\mathfrak{Q}_{4}\right)$. Also, by Theorem 4.2, $\varrho=\mu T^{-1}$ where $\mu \in M_{R}^{\sigma}\left(\Omega_{3}\right)$. Since $\mathfrak{L}_{1} \subset \mathfrak{L}_{3} \subset \tau \mathfrak{Q}_{1}$ and $\mathfrak{L}_{1}, \mathfrak{L}_{3}$ are $\delta$-lattices, using Theorem 3.2 , we have that $\mathscr{Q}_{3}$ is strongly measure replete. Then by the same argument as in the proof of Theorem 4.1, $\varrho=\mu T^{-1} \in M_{R\left(\mathcal{L}_{4}\right)}$. From which it follows, since $v=\left.\varrho\right|_{\mathfrak{2}\left(\Omega_{2}\right)}$ and since $\mathfrak{L}_{2} \subset \mathfrak{S}_{4}$, that $v \in M_{R}^{t}\left(\mathfrak{L}_{2}\right)$ and so $\mathfrak{L}_{2}$ is strongly measure replete.

Note. In general, it is easy to see that for lattices $\mathfrak{\Omega}_{2}, \mathfrak{\Omega}_{4}$ of $Y$ such that $\mathfrak{I}_{2} \subset \mathfrak{S}_{4}$, if $\mathfrak{I}_{4}$ is countably paracompact and if $\mathfrak{I}_{2}$ separates $\mathfrak{I}_{4}$, then $\mathfrak{I}_{4}$ is $\mathfrak{I}_{2}$ countably paracompact (cb). Thus the initial hypothesis of Theorem 4.3 concerning $\mathfrak{I}_{2}$ and $\mathfrak{I}_{4}$ will be satisfied in this case.

Now we can apply the previous theorems to the following cases:

(1) Let $X$ and $Y$ be $T_{3 \frac{1}{2}}$ topological spaces. Let $T: X \rightarrow Y$ be perfect. Take

$$
\begin{aligned}
& \mathfrak{I}_{1}=\mathfrak{J}_{X} \subset \mathfrak{I}_{X}=\mathfrak{I}_{3}=\tau \mathfrak{I}_{1}, \\
& \mathfrak{I}_{2}=\mathfrak{J}_{Y} \subset \mathfrak{I}_{Y}=\mathfrak{I}_{4}=\tau \mathfrak{I}_{2} .
\end{aligned}
$$

If $Y$ is countably paracompact and normal, then $X$ strongly measure compact implies that $Y$ is strongly measure compact.

(2) Let $X$ and $Y$ be topological spaces, and $T: X \rightarrow Y$ be perfect. Take

$$
\begin{aligned}
& \mathfrak{I}_{1}=\mathfrak{I}_{X}=\mathfrak{I}_{3}=\tau \mathfrak{I}_{1}, \\
& \mathfrak{I}_{2}=\mathfrak{I}_{Y}=\mathfrak{I}_{4}=\tau \mathfrak{I}_{2} .
\end{aligned}
$$

Assume that $Y$ is countably paracompact and normal. Then $\mathfrak{J}_{X}$ strongly measure replete implies $\mathfrak{J}_{Y}$ strongly measure replete.

THEOREM 4.4. Let $T: X \rightarrow Y$ be bijective and let $\mathfrak{L}_{1}$ and $\mathfrak{\Omega}_{2}$ be $\delta$-lattices of subsets of $X$ and $Y$, respectively. Let $T$ be $\mathfrak{L}_{1}-\mathfrak{I}_{2}$ continuous and $\sigma\left(\mathfrak{L}_{1}\right)-\sigma\left(\mathfrak{L}_{2}\right)$ closed. Then $\mathfrak{L}_{1}$ strongly measure replete implies $\mathfrak{S}_{2}$ strongly measure replete.

Proof. We may identify $X$ and $Y$ (via the map $x \rightarrow T x$ ). Then $\mathfrak{I}_{2} \subset \mathfrak{I}_{1}$, and $\sigma\left(\mathfrak{S}_{2}\right)=\sigma\left(\mathfrak{S}_{1}\right)$. The result now follows directly from Theorem 3.3, part (a).

The following example is an immediate application of Theorem 4.4.

EXAMPLE (Moran [12]). Let $X$ and $Y$ be $T_{3 \frac{1}{2}}$ topological spaces, and let $T: X \rightarrow Y$ be continuous and bijective such that $T^{-1}$ is Baire measurable. We take $\mathfrak{I}_{1}=\mathfrak{3}_{X}, \mathfrak{L}_{2}=\mathfrak{3}_{Y}$ and recall that $T$ continuous implies $T$ is $\mathfrak{3}_{X}-\mathfrak{3}_{Y}$ continuous. Then $X$ strongly measure compact implies $Y$ strongly measure compact.

LEMMA 4.1. Let $\mathfrak{\Omega}_{1}$ and $\mathfrak{S}_{2}$ be lattices of subsets of $X$ and $Y$, respectively and let $T: X \rightarrow Y$ be $\mathfrak{I}_{1}-\mathfrak{L}_{2}$ closed. Then given any $S \subset Y$ and any $L_{1}^{\prime} \in \mathfrak{L}_{1}^{\prime}$ such that $T^{-1}(S) \subset L_{1}^{\prime}$, there exists $L_{2}^{\prime} \in \mathfrak{\Omega}_{2}^{\prime}$ such that $S \subset L_{2}^{\prime}$ and $T^{-1}\left(L_{2}^{\prime}\right) \subset L_{1}^{\prime}$.

Proof. Omitted.

LEMMA 4.2. Let $\mathfrak{I}_{1}$ and $\mathfrak{I}_{2}$ be lattices of subsets of $X$ and $Y$, respectively and let $T: X \rightarrow Y$ be $\mathfrak{I}_{1}-\mathfrak{I}_{2}$ continuous and $\mathfrak{L}_{1}-\tau \mathfrak{\Omega}_{2}$ closed such that $T^{-1} y$ is $\mathfrak{L}_{1}$ compact for each $y \in Y$. Let $K_{2}$ be $\mathfrak{L}_{2}$-compact, then $T^{-1}\left(K_{2}\right)$ is $\mathfrak{Q}_{1}$-compact.
Proof. Let $K_{1}=T^{-1}\left(K_{2}\right)$ and suppose $K_{1} \subset \bigcup L_{1, \alpha}^{\prime} \in \mathfrak{I}_{1}$, then for each $y \in K_{2}$, there exists a finite subcollection of the $\left\{L_{1, \alpha}^{\prime}\right\}^{\alpha}=H_{y}$ which covers $T^{-1} y$. Let $\hat{L}_{y}^{\prime}=\bigcup H_{y} \in \mathfrak{\Omega}_{1}^{\prime}$, so $T^{-1} y \subset \hat{L}_{y}^{\prime}$. Since $T$ is $\mathfrak{\Omega}_{1}-\tau \mathfrak{\Omega}_{2}$ closed, there exists $O_{2, y}$ such that $y \in O_{2, y} \in\left(\tau L_{2}\right)^{\prime}$ and $T^{-1}\left(O_{2, y}\right) \subset \hat{L}_{y}^{\prime}$ (by Lemma 4.1). Now $K_{2} \subset \bigcup_{y} O_{2, y}$. Since $K_{2}$ is $\mathfrak{I}_{2}$-compact, it is $\tau \mathfrak{\Omega}_{2}$-compact; therefore $K_{2} \subset \bigcup_{i=1}^{n} O_{2, y_{i}}$. Therefore $K_{1}=T^{-1}\left(K_{2}\right) \subset \bigcup_{i=1}^{n} T^{-1}\left(O_{2, y_{i}}\right) \subset \bigcup_{1}^{n} \hat{L}_{y_{i}}^{\prime}$. So the collection $\left\{\hat{L}_{y_{i}}^{\prime}\right\}_{i=1, \ldots, n}$ covers $K_{1}$ and since $\hat{L}_{y_{t}}^{\prime}=\bigcup H_{y_{t}}, H=H_{y_{1}} \cup \ldots \cup H_{y_{n}}$ covers $T^{-1}\left(K_{2}\right)=K_{1}$, and each $H_{y_{t}}$ consists of only a finite number of elements of a given covering. Hence $T^{-1}\left(K_{2}\right)$ is $\mathfrak{I}_{1}$-compact.

THEOREM 4.5. Let $\mathfrak{\Omega}_{1}$ and $\mathfrak{\Omega}_{2}$ be $\delta$-lattices of $X$ and $Y$ respectively and $\sigma\left(\mathfrak{\Omega}_{2}\right)$ $\subset s\left(\mathfrak{I}_{2}\right)$. Let $T: X \rightarrow Y$ be $\mathfrak{\Omega}_{1}-\mathfrak{\Omega}_{2}$ continuous and $\mathfrak{\Omega}_{1}-\tau \mathfrak{\Omega}_{2}$ closed such that $T^{-1} y$ is $\mathfrak{L}_{1}$-compact for each $y \in Y$. Then $\mathfrak{L}_{2}$ strongly measure replete implies $\mathfrak{L}_{1}$ strongly measure replete.

Proof. Let $\mu \in M_{R}^{\sigma}\left(\mathfrak{\Omega}_{1}\right)$, then $v=\mu T^{-1} \in M_{R}^{\sigma}\left(\mathfrak{I}_{2}\right)$ since $\mathfrak{\Omega}_{2}$ is $\delta$ and $\dot{\sigma}\left(\mathfrak{\Omega}_{2}\right)$ $c s\left(\mathfrak{S}_{2}\right)$, so $v \in M_{R}^{t}\left(\mathfrak{Q}_{2}\right)$ since $\mathfrak{\Omega}_{2}$ is strongly measure replete. Therefore there exists $K_{2} \in \Omega_{2}=\Omega_{2}$-compact sets such that

$$
\nu^{*}\left(K_{2}\right) \geqslant \nu(Y)-\varepsilon=\mu(X)-\varepsilon .
$$

Now $K_{1}=T^{-1}\left(K_{2}\right)$ is $\mathfrak{L}_{1}$-compact by Lemma 4.2 , and

$$
\mu^{*}\left(K_{1}\right)=\inf \mu\left(L_{1}^{\prime}\right) \quad \text { where } K_{1} \subset L_{1}^{\prime}, L_{1} \in \mathfrak{I}_{1} .
$$

Let $K_{1} \subset L_{1}^{\prime}, L_{1} \in \mathfrak{I}_{1}$, so $K_{1} \cap L_{1}=\varnothing$ or $T^{-1}\left(K_{2}\right) \cap L_{1}=\varnothing$. Therefore $K_{2} \cap T L_{1}$ $=\varnothing$ : for if $y \in K_{2} \cap T L_{1}$, then $y \in K_{2}$ and $y=T x, x \in L_{1}$, and $T x \in K_{2}$, so $x \in T^{-1}\left(K_{2}\right) \cap L_{1}$, a contradiction. Hence $K_{2} \subset\left(T L_{1}\right)^{\prime} \in\left(\tau \mathfrak{\Omega}_{2}\right)^{\prime}$. Now if we let $T L_{1}=\bigcap L_{2, \alpha},\left(T L_{1}\right)^{\prime}=\bigcup L_{2, \alpha}^{\prime}$, then $K_{2} \subset \bigcup_{i=1}^{n} L_{2, \alpha_{i}}^{\prime} \subset\left(T L_{1}\right)^{\prime}$ or $K_{2} \subset L_{2}^{\prime}=\bigcup_{i=1}^{n} L_{2, \alpha_{i}}^{\prime}$ $\subset\left(T L_{1}\right)^{\prime}, \tilde{L}_{2}^{\prime} \in \mathfrak{L}_{2}^{\prime}$. Therefore $v^{*}\left(K_{2}\right) \leqslant v\left(\tilde{L}_{2}^{\prime}\right) \leqslant v^{*}\left(\left(T L_{1}\right)^{\prime}\right)$. But $L_{1} \subset T^{-1}\left(T L_{1}\right)^{\prime}$; therefore $L_{1}^{\prime} \supset\left(T^{-1}\left(T L_{1}\right)\right)^{\prime}=T^{-1}\left(\left(T L_{1}\right)^{\prime}\right) \supset T^{-1}\left(\tilde{L}_{2}^{\prime}\right)$. So $\mu\left(L_{1}^{\prime}\right) \geqslant \mu T^{-1}\left(\tilde{L}_{2}^{\prime}\right)=v\left(\tilde{L}_{2}^{\prime}\right)$ $\geqslant \nu^{*}\left(K_{2}\right)$ and $L_{1}^{\prime} \supset K_{1}$. Therefore $\mu^{*}\left(K_{1}\right) \geqslant \nu^{*}\left(K_{2}\right) \geqslant \mu(X)-\varepsilon$, hence $\mu \in M_{R}^{t}\left(\Omega_{1}\right)$. Thus $\mathfrak{I}_{1}$ is strongly measure replete.

Note. Theorem 4.5 is also true if $T$ is surjective and if instead of assuming $\mathbb{L}_{2}$ to be $\delta$ and $\sigma\left(\mathfrak{\Omega}_{2}\right) \subset s\left(\mathfrak{\Omega}_{2}\right)$, assume $T^{-1}\left(\mathfrak{I}_{2}\right)$ semi-separates $\mathfrak{\Omega}_{1}$, for then $v \in M_{R}^{\sigma}\left(\mathfrak{L}_{2}\right)$ also and the same proof follows. This will be the case if $T$ is $\Omega_{1}-\mathfrak{L}_{2}$ closed.

The following two examples are applications of Theorem 4.5:

(1) Let $T: X \rightarrow Y$ be continuous, perfect and 3 -closed, where $X$ and $Y$ are $T_{31}$ spaces. Then $Y$ strongly measure compact implies $X$ strongly measure compact.

(2) Let $X$ and $Y$ be topological spaces. Let $T: X \rightarrow Y$ be perfect. Then using the note following Theorem $4.5, \mathfrak{I}_{Y}$ strongly measure replete implies $\mathfrak{J}_{X}$ strongly measure replete. 
Corollary. Let $E \subset X$. Let $\mathfrak{I}_{E}$ be a $\delta$-lattice of subsets of $E$ such that $\mathfrak{I} \cap E$ $\subset \mathfrak{L}_{E}$ where $\mathfrak{L}$ is a $\delta$ lattice of subsets of $X$. If $\sigma(\mathfrak{L}) \subset s(\mathfrak{L})$ and if $\mathfrak{I}_{E} \subset \tau \mathfrak{I}$, then $\mathfrak{L}$ strongly measure replete implies $\mathfrak{\Omega}_{E}$ strongly measure replete.

Proof. The injection map $i: E \rightarrow X$ is $\mathfrak{Q}_{E}-\mathfrak{L}$ continuous and $i$ is $\mathfrak{L}_{E}-\tau \mathfrak{Q}$ closed. Also for $y \in X, i^{-1} y$ is $\mathfrak{L}_{E}$-compact and $\sigma(\mathfrak{L}) \subset s(\mathfrak{L})$. Therefore by Theorem $4.5, \mathfrak{I}$ strongly measure replete implies $\mathfrak{L}_{E}$ strongly measure replete.

We also have immediate application for the above corollary:

(1) Let $X$ be $T_{3 \frac{1}{2}}$. Take $\mathfrak{L}=\mathfrak{3}_{X}, \mathfrak{L}_{E}=\mathfrak{3}_{E}, E \in \tau \mathfrak{L}$. Then $\mathfrak{L} \cap E=\mathfrak{3} \cap E$ $\subset \mathcal{Z}_{E}$, and if $W \in \mathcal{Z}_{E}=\mathfrak{Q}_{E}$, then $W=F \cap E, F, E \in \tau \mathscr{Q}$, so $W \in \tau \mathfrak{Q}$. Therefore $\mathfrak{L}_{E} \subset \tau \mathcal{L}$. And of course $\sigma(\mathfrak{L}) \subset s(\mathfrak{L})$, hence by the corollary, any closed set in a strongly measure compact space is strongly measure compact.

(2) Let $X$ be a topological space. Take $\mathfrak{L}=\mathfrak{I}_{X}, E \in \mathfrak{I}$ and $\mathfrak{I}_{E}=\mathfrak{I}_{E}$. Then $\mathfrak{J}_{X} \cap E=\mathfrak{I}_{E}$, and if $W \in \mathfrak{I}_{E}$, then $W \in \tau \mathscr{Q}$, so $\mathfrak{I}_{E} \subset \tau \mathfrak{Q}$. If $\sigma\left(\mathfrak{I}_{X}\right) \subset s\left(\mathfrak{I}_{X}\right)$, using the corollary, we have any closed set $E$ in a topological space $X$ such that $\sigma\left(\mathfrak{I}_{X}\right) \subset s\left(\mathfrak{J}_{X}\right)$ and such that $\mathfrak{I}_{X}$ is strongly measure replete, if $\mathfrak{J}_{E}$ is strongly measure replete.

5. The remainder ${ }^{i}(\mathscr{L})-X$. In this section we initiate a study of the general remainder $I_{R}(\mathfrak{L})-X$. To each $\mu \in M_{R}(\mathfrak{I})$ we associate (see below) two measures $\tilde{\mu}$ and $\hat{\mu}$ defined on certain algebras of subsets of $I_{R}(\mathfrak{L})$. In terms of these measures we then get useful criteria for when $\mu$ is also $\sigma$-smooth, $\tau$-smooth or tight. This work generalizes the work of [4] where it was necessary to assume that $\mathfrak{L}$ was $\delta$-normal in order to utilize the Alexandroff Representation Theorem [1]. Here we only assume $\mathfrak{L}$ is separating and disjunctive. Even the separating condition is not critical for all theorems if one replaces $X$ by its image in $I_{R}(\mathscr{L})$ under the map $x \rightarrow \mu_{x}$. Getting rid of the assumption of $\delta$ and normal enables us to consider together remainders such as $\omega X-X$, where $\omega X$ is the Wallman compactification of $X$ [17], $\beta_{0} X-X$, where $\beta_{0} X$ is the Banaschewski compactification of $X$ (see [6]), in addition to of course $\beta X-X$. Our work here, therefore, substantially generalizes [4] which itself generalized the work of [11] and [9] where only the special top: ological case of $X$ a Tychonoff space, and $\mathfrak{L}=3_{X}=$ the lattice of zero sets was considered:

Let $\mathfrak{L}$ be separating and disjunctive.

If $A \in \mathfrak{Q}(\mathfrak{L})$, let $W(A)=\left\{\mu \in I_{\mathbb{R}}(\mathfrak{L}) \mid \mu(A)=1\right\}$, clearly for $A, B \in \mathfrak{U}(\mathfrak{L})$, we have the following properties:

(1) $W(A \cup B)=W(A) \cup W(B)$

(2) $W(A \cap B)=W(A) \cap W(B)$

(3) $W\left(A^{\prime}\right)=W(A)^{\prime}$,

(4) $\mathfrak{A}(W(\mathfrak{L}))=W(\mathfrak{A}(\mathfrak{L}))$,

(5) $A \supset B$ if only if $W(A) \supset W(B)$.

Now let $\mu \in M_{R}(\mathscr{L})$, define

$$
\hat{\mu}(W(A))=\mu(A) .
$$

Then it is easy to see $\hat{\mu} \in M_{R}(W(\mathfrak{I}))$ and conversely if $v \in M_{R}(W(\mathfrak{L}))$, define for $A \in \mathfrak{U}(\mathfrak{L})$,

$$
\mu(A)=v(W(A))
$$

then $\mu \in M_{R}(\mathfrak{L})$ and $v=\hat{\mu}$.

We note that since $W(\mathfrak{L})$ is a compact lattice, $M_{R}(W(\mathfrak{I}))=M_{R}^{\alpha}(W(\mathfrak{I}))$ $=M_{R}^{\tau}(W(\mathfrak{L}))=M_{R}^{t}(W(\mathfrak{L}))$.

(a) $\sigma$-Smooth.

THEOREM 5.1. Let $\mathfrak{I}$ be a lattice of subsets of an abstract set $X$. Let $\mathfrak{L}$ be separating and disjunctive, and $\mu \in M_{\mathbb{R}}(\mathfrak{L})$. Then the following are equivalent:

(1) $\mu \in M_{R}^{\sigma}(\mathfrak{I})$.

(2) $\hat{\mu}\left(\bigcap_{1}^{\infty} W\left(L_{i}\right)\right)=0, \bigcap_{1}^{\infty} W\left(L_{i}\right) \subset I_{R}(\mathfrak{I})-X, L_{i} \downarrow, L_{i} \in \mathfrak{L}$.

(3) $\hat{\mu}\left(\bigcap_{1}^{\infty} W\left(L_{i}\right)\right)=0, \bigcap_{1}^{\infty} W\left(L_{i}\right) \subset I_{R}(\mathbb{L})-I_{R}^{\sigma}(\mathbb{L}), L_{i} \downarrow, L_{i} \in \mathfrak{L}$.

(4) $\hat{\mu}^{*}(X)=\hat{\mu}\left(I_{R}(\mathfrak{I})\right)$.

Proof. (1) $\Rightarrow$ (2). Suppose $\mu \in M_{R}^{\sigma}(\mathfrak{L})$ and suppose $\bigcap_{1}^{\infty} W\left(L_{i}\right) \subset I_{R}(\mathfrak{L})-X$, $L_{i} \downarrow, L_{i} \in \mathfrak{L}$. Then intersecting both sides with $X$, we get $\bigcap_{1}^{\infty} L_{i}=\varnothing$, so $\mu\left(L_{i}\right) \rightarrow 0$. Now $\hat{\mu}\left(\bigcap_{1}^{\infty} W\left(L_{i}\right)\right)=\lim \hat{\mu}\left(W\left(L_{i}\right)\right)=\lim \mu\left(L_{i}\right)=0$.

$(2) \Rightarrow(1)$. Let $L_{i} \downarrow \varnothing$. If $\mu_{x} \in \bigcap_{1}^{\infty} W\left(L_{i}\right)$, then $\mu_{x} \in W\left(L_{i}\right)$ for all $i$ and $\mu_{x}\left(L_{i}\right)=1$ for all $i$, and so $\mu_{x}\left(\bigcap_{1}^{\infty} L_{i}\right)=1$; hence $x \in \bigcap_{1}^{\infty} L_{i}=\varnothing$, a contradiction. Therefore $\bigcap_{1}^{\infty} W\left(L_{i}\right) \subset I_{R}(\mathfrak{L})-X$ and by hypothesis, $\hat{\mu}\left(\bigcap_{1}^{\infty} W\left(L_{i}\right)\right)=0$. So $\lim \hat{\mu}\left(W\left(L_{i}\right)\right)=0$ where $\hat{\mu}\left(W\left(L_{i}\right)\right)=\mu\left(L_{i}\right)$; therefore $\mu \in M_{R}^{\sigma}(\mathfrak{L})$.

$(1) \Rightarrow$ (3). Let $\mu \in M_{R}^{\sigma}(\mathfrak{L})$. Suppose $\bigcap_{1}^{\infty} W\left(L_{i}\right) \subset I_{R}(\mathfrak{I})-I_{R}^{\sigma}(\mathfrak{I}), L_{i} \downarrow, L_{i} \in \mathfrak{I}$. Then $\bigcap_{1}^{\infty} W\left(L_{i}\right) \subset I_{R}(\mathfrak{L})-I_{R}^{\sigma}(\mathfrak{L}) \subset I_{R}(\mathfrak{L})-X$ and by $(2), \hat{\mu}\left(\bigcap_{1}^{\infty} W\left(L_{i}\right)\right)=0$.

(3) $\Rightarrow$ (1). Let $L_{i} \downarrow \varnothing$. If $v \in \bigcap_{1}^{\infty} W\left(L_{i}\right), v \in I_{\mathbf{R}}^{\sigma}(\mathbb{Q})$, then $v\left(L_{i}\right)=1$ for all $i$ and $v\left(\bigcap_{1}^{\infty} L_{i}\right)=1$, a contradiction since $v \in I_{R}^{\sigma}(\mathscr{\Omega})$, and $\bigcap_{1}^{\infty} L_{i}=\varnothing$. Therefore $\bigcap_{1}^{\infty} W\left(L_{i}\right)$ $\subset I_{R}(\mathscr{R})-I_{R}^{\sigma}(\mathscr{I})$ and so by assumption $\hat{\mu}\left(\bigcap_{1}^{\infty} W\left(L_{i}\right)\right)=0$. Hence $\hat{\mu}\left(W\left(L_{i}\right)\right)=0$ where $\hat{\mu}\left(W\left(L_{i}\right)\right)=\mu\left(L_{i}\right), L_{i} \downarrow \varnothing, L_{i} \in \mathfrak{S}$. Therefore $\mu \in M_{\mathrm{R}}^{\sigma}(\mathfrak{L})$. 
$(2) \Leftrightarrow(4) . \hat{\mu}_{*}\left(I_{R}(\Omega)-X\right)=\sup \hat{\mu}\left(\bigcap_{1}^{\infty} W\left(L_{i}\right)\right) \quad\left(\right.$ where $\left.\bigcap_{1}^{\infty} W\left(L_{i}\right) \subset I_{R}(\Omega)-X\right)=0$ if (2) holds. But $\hat{\mu}_{*}\left(I_{R}(\mathfrak{L})-X\right)+\hat{\mu}^{*}(X)=\hat{\mu}^{*}\left(I_{R}(\mathfrak{L})\right)$. Therefore $\hat{\mu}^{*}(X)=\hat{\mu}\left(I_{R}(\mathfrak{L})\right)$ and conversely.

Note. Let $\mu \in M_{R}(\mathfrak{L})$. Define $\mu^{\prime}$ on $\mathfrak{A}\left(W_{\sigma}(\mathfrak{L})\right)=W_{\sigma}(\mathfrak{A}(\mathfrak{L}))$ by

$$
\mu^{\prime}\left(W_{\sigma}(B)\right)=\mu(B), \quad B \in \mathfrak{A}(\mathfrak{L}),
$$

where $W_{\sigma}(A)=\left\{\mu \in I_{\mathbb{R}}^{\sigma}(\mathfrak{L}) \mid \mu(A)=1\right\}, \quad A \in \mathfrak{Q}(\mathfrak{I})$. Then it can be shown that $\mu^{\prime} \in M_{R}\left(W_{\sigma}(\mathfrak{L})\right)$, and conversely if $\varrho \in M_{R}\left(W_{\sigma}(\mathfrak{L})\right)$; then $\varrho=\mu^{\prime}, \quad \mu \in M_{R}(\mathfrak{I})$. Furthermore, we have the following theorem:

THEOREM 5.2. If $\mu \in M_{R}(\mathfrak{I})$, then $\mu \in M_{R}^{\sigma}(\mathfrak{I})$ iff $\mu^{\prime} \in M_{R}^{\sigma}\left(W_{\sigma}(\mathfrak{R})\right)$.

Proof. If $\mu \in M_{R}^{\sigma}(\mathfrak{L})$ and if $W_{\sigma}\left(L_{n}\right) \downarrow \varnothing, L_{n} \in \mathfrak{L}$, then $\bigcap_{1}^{\infty} W_{\sigma}\left(L_{n}\right)=\varnothing$ where $\bigcap_{1}^{\infty} W_{\sigma}\left(L_{n}\right)=W_{\sigma}\left(\bigcap_{1}^{\infty} L_{n}\right)$, so $L_{n} \downarrow \varnothing$. Since $\mu \in M_{R}^{\sigma}(\mathfrak{L}), \quad \mu^{\prime}\left(W_{\sigma}\left(L_{n}\right)\right)=\mu\left(L_{n}\right) \rightarrow 0$. Therefore $\mu^{\prime} \in M_{R}^{\sigma}\left(W_{\sigma}(\mathscr{L})\right)$. Conversely if $\mu^{\prime} \in M_{R}^{\sigma}\left(W_{\sigma}(\mathscr{I})\right)$, and if $L_{n} \downarrow \varnothing$, then $W_{\sigma}\left(L_{n}\right) \downarrow \varnothing$, and $\mu\left(L_{n}\right)=\mu^{\prime}\left(W_{\sigma}\left(L_{n}\right)\right) \rightarrow 0$. Therefore $\mu \in M_{R}^{\sigma}(\mathfrak{I})$.

COROLlary. Let $\mathfrak{L}$ be a separating and disjunctive lattice of subsets of an abstract set $X$. Then $I_{R}^{\sigma}(\mathfrak{I})$ is $W_{\sigma}(\mathfrak{L})$-replete.

Proof. Let $\mu^{\prime} \in I_{R}^{\sigma}\left(W_{\sigma}(\mathscr{I})\right)$. Then the associated $\mu \in I_{R}^{\sigma}(\mathfrak{L})$ and conversely by Theorem 5.2. Now $S\left(\mu^{\prime}\right)=\bigcap W_{\sigma}(L)$ where $\mu^{\prime}\left(W_{\sigma}(L)\right)=1, L \in \mathfrak{L}$. But $\mu^{\prime}\left(W_{\sigma}(L)\right)=1 \Leftrightarrow \mu(L)=1 \Leftrightarrow \mu \in W_{\sigma}(L)$. Therefore $\mu \in S\left(\mu^{\prime}\right)$, so $I_{R}^{\sigma}(\mathscr{I})$ is $W_{\sigma}(\mathfrak{L})$-replete.

THEOREM 5.3. If $\varrho \in M_{R}^{\sigma}(\delta W(\mathscr{L}))$ and if $\varrho^{*}(X)=\varrho\left(I_{R}(\mathfrak{Q})\right)$ then $\varrho=\hat{\mu}$ where $\mu \in M_{R}^{\sigma}(\mathfrak{L})$.

Proof. Since $\varrho \in M_{R}^{\sigma}(\delta W(\mathfrak{L})), \varrho$ is defined on $\mathfrak{X}(\delta W(\mathscr{I}))$. Consider the restriction of $\varrho$ to $\mathfrak{A}(W(\mathfrak{L}))$ and denote it by $\varrho$ again. Then $\varrho \in M_{R}^{\sigma}(W(\mathfrak{L}))$. since $W(\mathfrak{E})$ separates $\delta W(\mathfrak{L})$ because $W(\mathfrak{L})$ is a compact lattice. Thus $\varrho=\hat{\mu}, \mu \in M_{R}(\mathfrak{L})$. Consequently, $\hat{\mu}^{*}(X)=\hat{\mu}\left(I_{R}(\mathscr{I})\right)$ and therefore $\mu \in M_{R}^{\sigma}(\mathbb{I})$ by Theorem 5.1.

Now we will tie in some of these results with those in [4].

THEOREM 5.4. Let $\mathfrak{I}$ be a separating and disjunctive lattice of subsets of an abstract set $X$. (1) If $\mathfrak{L}$ is $\delta$-normal, then $\mathfrak{Z}(\tau W(\mathfrak{I})) \subset \delta W(\mathfrak{Z}(\mathfrak{L}))$. (2) If $\mathfrak{L}$ is normal and countably paracompact, and if $\bigcap_{1}^{\infty} W\left(L_{n}\right) \subset I_{R}(\mathfrak{S})-X, L_{n} \in \mathfrak{L}$ then there exists $K_{0} \in \mathcal{Z}(\tau W(\mathfrak{I}))$ such that $\bigcap_{1}^{\infty} W\left(L_{n}\right) \subset K_{0} \subset I_{R}(\mathfrak{L})-X$, and if $\mathfrak{L}$ is also $\delta ; K_{0}$ $=\cap W\left(\hat{L}_{n}\right), \hat{L}_{n} \in \mathcal{Z}(\mathbb{L})$.

Proof. (1). If $\mathfrak{L}$ is separating, disjunctive and $\delta$-normal, then $C_{b}(\mathfrak{R})$ $=C\left(I_{R}(\mathfrak{I})\right)$ (in the sense of $f \rightarrow \hat{f}$ where $f \in C_{b}(\mathfrak{L})$ and $\int f d \mu=\hat{f}(\mu) \in C\left(I_{R}\left(\mathfrak{Q}_{n}\right)\right)$ (see [4]). Suppose $K_{0} \in \mathfrak{3}(\tau W(\mathfrak{L}))$, then $K_{0}=\hat{f}^{-1}(0), f \in C_{b}(\mathfrak{I})$, and $K_{0}^{\text {r }}$ $=\bigcap_{1}^{\infty}\left\{\mu \in I_{R}(\mathfrak{L})|\quad| \hat{f}(\mu) \mid \leqslant 1 / n\right\}$. Let $K_{n}=\left\{\mu \in I_{R}(\mathfrak{L})|\quad| \hat{f}(\mu) \mid \leqslant 1 / n\right\}$. Then $K_{n}$ $\in \mathcal{Z}^{1}(\tau W(\mathscr{L}))$, and $K_{n} \cap X \in \mathcal{Z}(\mathfrak{L})$. Let $K_{n} \cap X=L_{n}=\{x \in X|\quad| f(x) \mid \leqslant 1 / n\}$, $L_{n} \in \mathfrak{L}, L_{n} \downarrow$. Then $K_{n} \supset L_{n}$, and so $K_{n} \supset W\left(L_{n}\right)=\bar{L}_{n}$. So $K_{0}=\cap K_{n} \supset \cap W\left(L_{n}\right)$. Now let $\mu \in K_{0}$, then $\vec{f}(\mu)=0$. Since $\bar{X}=W(X)=I_{R}(\mathscr{Q})$, there exists a net $\left\{\mu_{x_{\alpha}}\right\}$ such that $\mu_{x_{\alpha}} \rightarrow \mu$. Then $\vec{f}\left(\mu_{x_{\alpha}}\right) \rightarrow \vec{f}(\mu)=0$. Therefore for any $n$, there exists $\alpha_{0}$ such that for all $\alpha \geqslant \alpha_{0},\left|\hat{f}\left(\mu_{x_{\alpha}}\right)\right| \leqslant 1 / n$. Hence $\mu_{x_{\alpha}} \in L_{n} \subset W\left(L_{n}\right)$, and since $\mu_{x_{\alpha}} \rightarrow \mu, \mu \in W\left(L_{n}\right)$ for any $n$. Therefore $\mu \in \bigcap W\left(L_{n}\right)$, and $K_{0}=\bigcap W\left(L_{n}\right)$, where $L_{n} \in \mathcal{Z}(\mathfrak{L})$. Thus $K_{0} \in \delta W(\mathfrak{Z}(\mathfrak{L}))$ and therefore $3(\tau W(\mathfrak{L})) \subset \delta W(\mathfrak{Z}(\mathfrak{L}))$.

(2) Suppose $\mathfrak{L}$ is countably paracompact, and consider $\bigcap_{1}^{\infty} W\left(L_{n}\right), L_{n} \in \mathfrak{L}, L_{n} \downarrow$. Suppose $\bigcap_{1}^{\infty} W\left(L_{n}\right) \subset I_{R}(\mathfrak{L})-X$, then as we know $\cap L_{n}=\varnothing$. Therefore by countably paracompact, there exists $\tilde{L}_{n} \in \mathfrak{L}$ such that $L_{n} \subset \tilde{L}_{n}^{\prime}$ and $\cap \tilde{L}_{n}^{\prime}=\varnothing$. So $\bigcap_{1}^{\infty} W\left(\tilde{L}_{n}\right)^{\prime} \subset I_{R}(\mathfrak{S})-X$. Now since $L_{n} \subset \tilde{L}_{n}^{\prime}$, we have $W\left(L_{n}\right) \subset W\left(\tilde{L}_{n}^{\prime}\right)=W\left(\tilde{L}_{n}\right)^{\prime}$, therefore $\bigcap_{1}^{\infty} W\left(L_{n}\right) \subset \bigcap_{1}^{\infty} W\left(\tilde{L}_{n}\right)^{\prime} \subset I_{R}(\mathfrak{L})-X$.

If $\mathfrak{L}$ is normal, then $\left(I_{R}(\mathfrak{L}), \tau W(\mathfrak{L})\right)$ is compact and $T_{2}$.

Also $\bigcap_{1}^{\infty} W\left(L_{n}\right)$ is compact and $\bigcap_{1}^{\infty} W\left(L_{n}\right) \subset W\left(\tilde{L}_{n}\right)^{\prime}$, which is open for any $n$. Therefore there exists $K_{n}$, a compact $G_{\delta}$ set such that $\bigcap_{1}^{\infty} W\left(L_{n}\right) \subset K_{n} \subset W\left(\tilde{L}_{n}\right)^{\prime}$ (by Baire-Sandwich Theorem). Therefore $\bigcap_{1}^{\infty} W\left(L_{n}\right) \subset \bigcap_{1}^{\infty} K_{n}$ (where $\bigcap_{1}^{\infty} K_{n}$ is also compact $\left.G_{\delta}\right) \subset \bigcap_{1}^{\infty} W\left(\tilde{L}_{n}\right)^{\prime} \subset I_{R}(\mathscr{L})-X$. So $\bigcap_{1}^{\infty} W\left(L_{n}\right) \subset K_{0} \subset I_{R}(\mathfrak{L})-X$ where $K_{0}$ $=\bigcap_{1}^{\infty} K_{n}$ is compact $G_{\delta}{ }^{\prime}$ and therefore $\in 3(\tau W(\Omega))$

Note. Since for any lattice $\mathfrak{L}, W(\mathfrak{L})$ and consequently $\tau W(\mathfrak{L})$; is compact, it follows readily that, without any added assumptions as in Theorem 5:4, $3(\tau W(\mathfrak{I})) \subset \sigma(W(\mathfrak{L}))$ always holds.

THEOREM 5.5. If $\mathfrak{L}$ is separating, disjunctive, and $\delta$-normal and countably paracompact, and if $\mu \in M_{R}(\mathfrak{L})$, then $\mu \in M_{R}^{\sigma}(\mathfrak{L})$ if and only if $\hat{\mu}\left(K_{0}\right)=0$ for all $K_{0}$ $\subset I_{R}(\mathfrak{L})-X$, where $K_{0} \in 3(\tau W(\mathfrak{L}))$.

Proof. Suppose $\mu \in M_{R}^{\sigma}(\mathscr{I})$, then by Theorem 5.1, $\mu\left(\bigcap_{1}^{\infty} W\left(L_{n}\right)\right)=0$ whenever $\bigcap_{1}^{\infty} W\left(L_{n}\right) \subset I_{R}(\mathfrak{L})-X$ where $L_{n} \in \mathfrak{L}, L_{n} \downarrow$. By Theorem 5.4, any $K_{0} \in \mathfrak{Z}(\tau W(\mathfrak{L}))$ can. be written in the form $K_{0}=\bigcap_{1}^{\infty} W\left(L_{n}\right), L_{n} \in \mathbb{L}, L_{n} \downarrow$, and so if $K_{0} \subset I_{R}(\mathbb{R})-X$ it follows that $\hat{\mu}\left(K_{0}\right)=0$. 
Conversely, let $\mu \in M_{R}(\mathfrak{Q})$ and $\hat{\mu}\left(K_{0}\right)=0$ for all $K_{0} \in \mathcal{3}(\tau W(\mathscr{I})) ; K_{0}$ $\subset I_{R}(\mathfrak{I})-X$. Then by Theorem 5.4, $\hat{\mu}\left(\bigcap_{1}^{\infty} W\left(L_{n}\right)\right)=0$ where $\bigcap_{1}^{\infty} W\left(L_{n}\right) \subset I_{R}(\mathfrak{I})-X$, $L_{n} \in \mathfrak{L}$, and, by Theorem 5.1, $\mu \in M_{R}^{\sigma}(\mathfrak{L})$

Note. Theorem 5.5 is a generalization of a theorem of Knowles [11].

(b) $\tau-\mathrm{Smooth}$

THEOREM 5.6. Let $\mathfrak{L}$ be a disjunctive and separating lattice of subsets of an abstract set $X$, and let $\mu \in M_{R}(\mathfrak{I})$. Then the following are equivalent:

(1) $\mu \in M_{R}^{\tau}(\mathfrak{I})$.

(2) $\tilde{\mu}$ vanishes on every closed set of $I_{R}(\mathfrak{L})-X$, where $\tilde{\mu} \in M_{R}(\tau W(\mathfrak{L}))$ and $\tilde{\mu}$ is the unique extension of $\hat{\mu}$ to $\mathfrak{A}(\tau W(\mathfrak{L}))$.

(3) $\tilde{\mu}^{*}(X)=\tilde{\mu}\left(I_{R}(\mathfrak{I})\right)$.

Proof. To show (1) $\Rightarrow(2)$, we let $\mu \in M_{R}^{\tau}(\mathbb{R})$. Suppose $\bigcap_{\alpha} W\left(L_{\alpha}\right) \subset I_{R}(\mathfrak{L})-X$, $L_{\alpha} \in \mathfrak{L}, L_{\alpha} \downarrow$. Then clearly $\bigcap_{\alpha} \dot{L}_{\alpha}=\varnothing$, so $\mu\left(L_{\alpha}\right) \rightarrow 0$. Now we can extend (uniquely since $W(\mathfrak{L})$ separates $\tau W(\mathfrak{L})$ ) by our general extension (see Theorem 3.1) $\hat{A}$ to $\tilde{\mu} \in M_{R}(\tau W(\mathfrak{L}))$, so $\tilde{\mu}$ is defined on $\mathfrak{A}(\tau W(\mathfrak{L}))$.

Since $\tau W(\mathfrak{L})$ is compact and $\tilde{\mu} \in M_{R}^{\tau}(\tau W(\mathfrak{L}))$ clearly and since $\tau W(\mathfrak{L})$ is a $\delta$-lattice, by Theorem $2.1 \tilde{\mu}\left(\bigcap W\left(L_{\alpha}\right)\right)=\lim _{\alpha} \hat{\mu}\left(W\left(L_{\alpha}\right)\right)=\lim _{\alpha} \mu\left(L_{\alpha}\right)=0$ if ก $W\left(L_{\alpha}\right) \subset I_{R}(\mathfrak{L})-X$. It follows that $\tilde{\mu}$ vanishes on every closed set of $I_{R}(\mathfrak{I})-X$. Conversely, to show $(2) \Rightarrow(1)$, we suppose that $L_{\alpha} \downarrow \varnothing, L_{\alpha} \in \mathcal{L}$. Then $\cap W\left(L_{\alpha}\right)$ $\subset I_{R}(\mathscr{Q})-X$, and so $\tilde{\mu}\left(\bigcap_{\alpha} W\left(L_{\alpha}\right)\right)=0$, where $\tilde{\mu}\left(\bigcap_{\alpha} W\left(L_{\alpha}\right)\right)=\lim _{\alpha} \tilde{\mu}\left(W\left(L_{\alpha}\right)\right)$ $=\lim _{\alpha} \mu\left(L_{\alpha}\right)$, therefore $\lim _{\alpha} \mu\left(L_{\alpha}\right)=0$, and so $\mu \in M_{R}^{\tau}(\mathscr{L})$.

The steps needed to show (2) $\Leftrightarrow$ (3) are similar to those shown in proving (2) $\Leftrightarrow(4)$ of Theorem 5.1.

Note. (A) If $\mathbb{L}$ is normal, then in Theorem $5.6,(2) \Leftrightarrow \tilde{\mu}$ vanishes on every compact set of $I_{R}(\mathfrak{I})-X$.

(B) If $\mathfrak{L}$ is not separating, we may work with the image of $X$ under the mapping $x \rightarrow \mu_{x}$ of $X$ in $I_{R}(\mathcal{Q})$, and Theorem 5.6 still holds.

THEOREM 5.7. Let $\mathcal{Q}$ be a separating and disjunctive lattice of subsets of an abstract set $X$. If $\mu \in M_{R}^{\tau}(\mathbb{I})$, then $\mu$ can be extended uniquely to $\gamma \in M_{R}^{\tau}(\tau \mathbb{R})$.

Proof. Let $\mu \in M_{R}^{\tau}(\mathscr{R})$. Then, from Theorem 5.6, we know that for every $K \in \tau W(\mathscr{L}), \quad K \subset I_{R}(\mathscr{L})-X, \quad \tilde{\mu}(K)=0$. Now $\quad \sigma(\tau W(\mathscr{I})) \cap X=\sigma(\tau W(\mathscr{L}) \cap X)$ $=\sigma(\tau \mathscr{Q})$. Define for $A \in \sigma(\tau \mathscr{\&})$,

$$
\gamma(A)=\tilde{\mu}\left(A^{*}\right) \text { where } A^{*} \cap X=A, A^{*} \in \sigma(\tau W(\mathscr{L})) .
$$

$\gamma$ is well defined since $X$ is $\tilde{\mu}$-thick in $I_{R}(\mathfrak{L})$ (Halmos [10]) by (3) of Theorem 5.6.
For $A \in \mathfrak{A}(\tau \mathfrak{L})$; since $\tilde{\mu}$ is $\tau W(\mathfrak{L})$-regular, for $\varepsilon>0$, there exists $K=\bigcap_{\alpha} W\left(L_{\alpha}\right)$ $\in \tau W(\mathfrak{I})$ such that

$$
\gamma(A)=\tilde{\mu}\left(A^{*}\right)<\tilde{\mu}(K)+\varepsilon=\tilde{\mu}\left(\bigcap_{\alpha} W\left(L_{\alpha}\right)\right)+\varepsilon, \quad A^{*} \supset K .
$$

By the definition of $\gamma, \gamma\left(\bigcap_{\alpha} L_{\alpha}\right)=\tilde{\mu}\left(\bigcap_{\alpha} W\left(L_{\alpha}\right)\right), \bigcap_{\alpha} L_{\alpha}=\bigcap_{\alpha} W\left(L_{\alpha}\right) \cap X$. So $\gamma(A)$ $<\gamma\left(\bigcap_{\alpha} L_{\alpha}\right)+\varepsilon, \bigcap_{\alpha} L_{\alpha} \in \tau \mathfrak{Q}, A^{\alpha} \supset \bigcap_{\alpha} L_{\alpha}$, and this shows that ${ }^{\alpha} \gamma$ is $\tau \mathscr{Q}$-regular. Now for $\mathscr{A}^{\alpha} \in \mathfrak{Q}(\mathfrak{L})$,

$$
\gamma(A)=\tilde{\mu}(W(A))=\hat{\mu}(W(A))=\mu(A) .
$$

Therefore $\gamma$ extends $\mu$.

Suppose $L_{\alpha} \downarrow \varnothing$. Then $\bigcap W\left(L_{\alpha}\right) \subset I_{R}(\mathfrak{R})-X$ and $\tilde{\mu}\left(\cap W\left(L_{\alpha}\right)\right)=0$.

Therefore $0=\gamma(\varnothing)=\gamma_{\alpha}\left(\bigcap_{\alpha} L_{\alpha}\right)=\gamma\left(\bigcap_{\alpha} W\left(L_{\alpha}\right) \cap X\right)=\tilde{\mu}\left(\bigcap_{\alpha} W\left(L_{\alpha}\right)\right)$ by the definition of $\gamma$.

Since $\tilde{\mu}$ is $\tau$-smooth and $\tau W(\mathscr{I})$ is a $\delta$-lattice, we have $\tilde{\mu}\left(\cap W\left(L_{\alpha}\right)\right)$ $=\lim _{\alpha} \tilde{\mu}\left(W\left(L_{\alpha}\right)\right)$ (by Theorem 2.1) $=\lim _{\alpha} \gamma\left(L_{\alpha}\right)$ by the definition of $\gamma$. Therefore $0=\lim _{\alpha} \gamma\left(L_{\alpha}\right)$ and it follows that $\gamma$ is $\tau$-smooth. The uniqueness part is elementary.

(c) Tight.

THEOREM 5.8. If $\mathfrak{I}$ is separating, disjunctive and normal, and if $\mu \in M_{R}(\mathfrak{I})$, then the following are equivalent:

(1) $\mu \in M_{R}^{t}(\mathbb{R})$,

(2) $X$ is $\tilde{\mu}^{*}$-measurable and $\tilde{\mu}^{*}(X)=\tilde{\mu}\left(I_{R}(\mathscr{Q})\right)$.

Proof. Let $\mu \in M_{R}^{t}(\mathfrak{S})$. Then for any $\varepsilon>0$ there exists an $\mathfrak{R}$-compact set $K$ such that $\mu_{*}\left(K^{\prime}\right)<\varepsilon$. Now $\mu \in M_{R}^{\tau}(\mathfrak{I})$ (since $M_{R}^{t}(\mathfrak{I}) \subset M_{R}^{\tau}(\mathfrak{I})$ by Theorem 2.3), so by Theorem 5.7, $\mu$ can be extended to $\gamma \in M_{R}^{\tau}(\tau \mathscr{S})$.

Also

$$
\mu^{*}(K)+\mu_{*}\left(K^{\prime}\right)=\mu(X)=\gamma(X) .
$$

$$
\begin{aligned}
\mu^{*}(K) & =\inf \mu(A), & & \text { where } K \subset A, A \in \sigma(\mathfrak{L}) \\
& =\inf \gamma(A), & & \text { where } K \subset A, A \in \sigma(\mathfrak{I}) \\
& \geqslant \gamma(K) . & &
\end{aligned}
$$

Therefore, $\mu^{*}(K) \geqslant \gamma(K)$.

Since $\mathfrak{L}$ is separating, disjunctive and normal (or since $\mathfrak{L}$ is $T_{2}$ ), $K \in \tau \mathfrak{L}$ and $K=\bigcap L_{\alpha}, L_{\alpha} \in \mathcal{L}, L_{\alpha} \downarrow$. Thus

$$
\begin{aligned}
\gamma(K) & =\gamma\left(\cap L_{\alpha}\right)=\inf \gamma\left(L_{\alpha}\right) \quad \text { (by Theorem 2.1) } \\
& \geqslant \inf \gamma(A), \quad \text { where } A \supset K, A \in \sigma(\mathcal{I}) \\
& =\mu^{*}(K) .
\end{aligned}
$$

Hence, $\gamma(K) \geqslant \mu^{*}(K)$. Consequently,

$$
\mu^{*}(K)=\gamma(K) \text { and } \quad \mu_{*}\left(K^{\prime}\right)=\gamma\left(K^{\prime}\right)
$$


Now $\varepsilon>\gamma\left(K^{\prime}\right)=\gamma(X-K)=\tilde{\mu}\left(I_{R}(\mathfrak{L})-K\right)$ (by the definition of $\gamma$ ). But, since $\mathscr{L}$ is normal, $I_{R}(\mathfrak{L})$ is compact $T_{2}$, therefore, $K \in \tau W(\mathfrak{L})$ so $I_{R}(\mathfrak{L})-X \subset I_{R}(\mathfrak{L})-K$ which is open, and it follows that $\tilde{\mu}^{*}\left(I_{R}(\mathfrak{I})-X\right)=0$. Hence, $I_{R}(\mathfrak{L})-X$ is $\tilde{\mu}^{*}$-measurable. Therefore, $X$ is $\tilde{\mu}^{*}$-measurable, and

$$
\tilde{\mu}^{*}(X)=\tilde{\mu}\left(I_{R}(\mathfrak{I})\right) \text {. }
$$

Conversely, suppose $X$ is $\tilde{\mu}^{*}$-measurable and $\tilde{\mu}^{*}(X)=\tilde{\mu}\left(I_{R}(\mathfrak{I})\right)$. Then by Theorem 5.6, $\mu \in M_{R}^{\tau}(\mathfrak{L})$. Since $\tilde{\mu}^{*}$ is $\tau W(\mathfrak{L})$-regular on $\tilde{\mu}^{*}$-measurable sets, there exists $K \in \tau W(\mathfrak{I}), K \subset X$ such that

$$
\tilde{\mu}(K)+\varepsilon>\tilde{\mu}^{*}(X)=\tilde{\mu}\left(I_{R}(\mathscr{L})\right)
$$

where $\varepsilon>0 . K$ is clearly $\mathfrak{L}$-compact and $K \in \tau \mathscr{L}$. Also, as above

$$
\mu^{*}(K)=\gamma(K)=\tilde{\mu}(K)
$$

Hence,

$$
\mu^{*}(K)+\varepsilon>\tilde{\mu}\left(I_{R}(\mathfrak{L})\right)=\mu(X) .
$$

Therefore, $\varepsilon>\mu(X)-\mu^{*}(K)=\mu_{*}\left(K^{\prime}\right)$ and so $\mu \in M_{R}^{*}(\mathbb{L})$. Note, in this part the normality of $\mathfrak{L}$ is not needed.

Definition. Let $\mathfrak{L}$ be a lattice of subsets of an abstract set $X$. $\mathfrak{L}$ is Øechcomplete if $I_{R}(\mathfrak{L})-X$ is an $F_{\sigma}$ set.

This is just a generalization of the usual topological notion (see [7])

THEOREM 5.9. If $\mathfrak{L}$ is separating, disjunctive, normal, Cech-complete, and Lindelöf, then $M_{R}^{\sigma}(\mathfrak{L})=M_{R}^{\mathfrak{\tau}}(\mathfrak{I})=M_{R}^{\mathrm{t}}(\mathfrak{L})$.

Proof. Since $\mathfrak{L}$ is Lindelöf, it follows that $M_{R}^{\sigma}(\mathfrak{L})=M_{R}^{\tau}(\mathfrak{L})$. Let $\mu \in M_{R}^{\sigma}(\mathfrak{L})$, then $\tilde{\mu}^{*}(X)=\tilde{\mu}\left(I_{R}(\mathbb{R})\right)$ (by Theorem 5.6). But since $\mathfrak{L}$ is Čech-complete,

$$
I_{R}(\mathfrak{L})-X \in F_{\sigma} \subset \sigma(\tau W(\mathfrak{L}))
$$

Therefore $X \in \sigma(\tau W(\mathfrak{L})) \subset \tilde{\mu}^{*}$-measurable sets. So by Theorem 5.8, $\mu \in M_{R}^{t}(\mathfrak{I})$. Hence $M_{R}^{\sigma}(\mathfrak{L}) \subset M_{R}^{t}(\mathfrak{L})$. Now $M_{R}^{t}(\mathfrak{L}) \subset M_{R}^{\sigma}(\mathfrak{L})$, therefore $M_{R}^{\sigma}(\mathfrak{L})=M_{R}^{t}(\mathfrak{L})$.

We give some applications of the last theorem.

(1) Let $X$ be a complete, separable metric space. Then $\mathfrak{I}_{X}=\mathfrak{3}_{X}$. Let $\mathfrak{I}=\mathfrak{I}_{X}$. Since $X$ is separable, $X$ is Lindelöf. Since $X$ is Čech-complete (see [7]) and since $\sigma\left(\mathfrak{Z}_{X}\right)=\varrho\left(\mathfrak{Z}_{X}\right)$, we get $M^{\sigma}(\mathfrak{I})=M_{R}^{\sigma}(\mathfrak{I})=M_{R}^{\mathfrak{t}}(\mathfrak{I})=M_{R}^{\mathrm{t}}(\mathfrak{I})$.

(2) Let $X$ be locally compact, $T_{2}$ and Lindelöf. Since $\mathfrak{I}=\mathfrak{I}_{X}$ is $\delta$, regular, and Lindelöf, $\mathfrak{I}$ is normal. Since $\mathfrak{I}$ is Čech-complete (see [7]) and since $\mathfrak{I}$ is disjunctive and separating, then applying Theorem 5.9 again, we have $M_{R}^{\sigma}(\mathfrak{I})$ $=M_{R}^{\mathfrak{\tau}}(\mathfrak{I})=M_{R}^{t}(\mathfrak{I})$.

Note. If $X$ is a locally compact, $T_{2}$, paracompact and separable space, then since paracompact and separable imply Lindelöf, we have again $M_{R}^{\sigma}(\mathfrak{I})=M_{R}^{\tau}(\mathfrak{I})$ $=M_{R}^{t}(\mathfrak{J})$.
6. Further applications. In this section we give some further applications of the general theorems of Section 5. We will only consider four particular topological lattices. We note in general that for $\mathfrak{L}$ separating and disjunctive, $W(L)=\bar{L}$. These four spaces are:

(1) $X$ is a $T_{1}$ space and $\mathfrak{L}=\mathfrak{I}=$ lattice of closed sets.

(2) $X$ is 0 -dimensional and $T_{2}, \mathfrak{L}=\mathbb{E}=$ clopen sets.

(3) $X$ is $T_{1}, \mathfrak{L}=\mathfrak{B}=$ Borel sets.

(4) $X$ is $T_{3 \frac{1}{2}}, \mathfrak{L}=\mathfrak{Z}=$ zero sets.

Applying Theorems 5.1 and 5.5 we get:

(1) Let $X$ be a $T_{1}$ topological space. Let $\mu \in M_{R}(\mathfrak{I})$, then the following are equivalent:

(i) $\mu \in M_{R}^{\sigma}(\Im)$.

(ii) $\mu\left(\bigcap_{1}^{\infty} \bar{F}_{i}\right)=0, \bigcap_{1}^{\infty} \bar{F}_{i} \subset \omega X-X\left(F_{i} \in \mathfrak{I}\right.$, closure in $\omega X$, the Wallman compactification).

(iii) $\hat{\mu}\left(\bigcap_{1}^{\infty} \bar{F}_{i}\right)=0, \cap \bar{F}_{i} \subset \omega X-I_{R}^{\sigma}(\mathfrak{J})$

(iv) $\hat{\mu}^{*}(X)=\hat{\mu}(\omega X)$.

By Theorem 5.5 , if $X$ is normal and countably paracompact, then $\mu \in M_{R}^{\sigma}(\mathfrak{I})$ iff $\hat{\mu}\left(K_{0}\right)=0$ for any zero set $K_{0}$ of $\omega X$ such that $K_{0} \subset \omega X-X$.

(2) Let $X$ be a 0 -dimensional and $T_{2}$ space. Let $\mu \in M_{R}(\mathbb{E})=M(\mathbb{E})$. Then the following are equivalent:

(i) $\mu \in M_{R}^{\sigma}(\mathbb{E})=M^{\sigma}$ (E) .

(ii) $\hat{\mu}\left(\bigcap_{1}^{\infty} \bar{C}_{i}\right)=0, \bigcap_{1}^{\infty} \bar{C}_{i} \subset \beta_{0} X-X\left(C_{i} \in \mathbb{E}\right.$, closure in $\beta_{0} X$, the Banaschewski compactification)

(iii) $\hat{\mu}\left(\bigcap_{1}^{\infty} \bar{C}_{i}\right)=0, \bigcap_{1}^{\infty} \bar{C}_{i} \subset \beta_{0} X-v_{0} X$, where $v_{0} X=I_{R}^{\sigma}(\mathfrak{E})=I^{\sigma}(\mathbb{E})$.

(iv) $\hat{\mu}^{*}(X)=\hat{\mu}\left(\beta_{0} X\right)$.

(3) Let $X$ be $T_{1}$. Let $\mu \in M_{R}(\mathfrak{B})=M(\mathfrak{B}), \mathfrak{B}=\sigma(\mathfrak{I})=$ Borel sets. Then the following are equivalent:

(i) $\mu \in M_{R}^{\sigma}(\mathfrak{B})=M^{\sigma}(\mathfrak{B})$

(ii) $\mu\left(\bigcap_{1}^{\infty} \bar{B}_{i}\right)=0, \bigcap_{1}^{\infty} \bar{B}_{i} \subset I_{R}(\mathfrak{B})-X=I(\mathfrak{B})-X$.

(iii) $\hat{\mu}\left(\bigcap_{1}^{\infty} \bar{B}_{i}\right)=0, \bigcap_{1}^{\infty} \bar{B}_{i} \subset I_{R}(\mathfrak{B})-I_{R}^{\sigma}(\mathfrak{B})=I(\mathfrak{B})-I^{\sigma}(\mathfrak{B})$.

(iv) $\hat{\mu}^{*}(X)=\hat{\mu}\left(I_{R}(\mathfrak{B})\right)$

By Theorem 5.5, $\mu \in M_{R}^{\sigma}(\mathfrak{B})=M^{\sigma}(\mathfrak{B})$ iff $\hat{\mu}\left(K_{0}\right)=0$ for any zero set $K_{0}$ of $I_{R}(\mathfrak{B})$ such that $K_{0} \subset I_{R}(\mathfrak{B})-X$.

(4) Let $X$ be a $T_{3,}$ topological space. Let $\mu \in M_{R}(3), 3=$ zero sets. Then the following are equivalent: 
(i) $\mu \in M_{R}^{\sigma}(3)$

(ii) $\hat{\mu}\left(\bigcap_{1}^{\infty} \bar{Z}_{i}\right)=0, \bigcap_{1}^{\infty} \bar{Z}_{i} \subset \beta X-X$.

(iii) $\hat{\mu}\left(\bigcap_{1}^{\infty} \bar{Z}_{i}\right)=0, \bigcap_{1}^{1} \bar{Z}_{i} \subset \beta X-v X$.

(iv) $\hat{\mu}^{*}(X)=\hat{\mu}(\beta X)$, where $\beta X=$ Stone-Čech compactification, and $\nu X=$ real compactification.

Also by Theorem $5.5, \mu \in M_{R}^{\sigma}(3)$ iff $\hat{\mu}\left(K_{0}\right)=0$ for any zero set $K_{0}$ of $\beta X$ such that $K_{0} \subset \beta X-X$.

Applying Theorem 5.6 to the same four spaces, we get:

(1) If $\mu \in M_{R}(\mathfrak{I})$, then $\mu \in M_{R}^{\tau}(\mathfrak{I}) \Leftrightarrow \tilde{\mu}$ vanishes on every closed set of $\omega X-X$ $\Leftrightarrow \tilde{\mu}^{*}(X)=\tilde{\mu}(\omega X)$.

(2) If $\mu \in M_{R}(\mathbb{E})=M(\mathbb{E})$, then $\mu \in M_{R}^{\tau}(\mathbb{E}) \Leftrightarrow \tilde{\mu}$ vanishes on every closed set of $\beta_{0} X-X$.

(3) If $\mu \in M_{R}(\mathfrak{B})=M(\mathfrak{B})$, then $\mu \in M_{R}^{\tau}(\mathfrak{B}) \Leftrightarrow \tilde{\mu}$ vanishes on every closed set of $I_{R}(\mathfrak{B})-X=I(\mathfrak{B})-X$.

(4) If $\mu \in M_{R}(3)$, then $\mu \in M_{R}^{\tau}(3) \Leftrightarrow \tilde{\mu}$ vanishes on every closed set of $\beta X-X$.

Finally if we apply Theorem.5.8 to the following spaces we have:

(1) If $X$ is $T_{4}$ and if $\mu \in M_{R}(\Im)$, then $\mu \in M_{R}^{t}(\Im)$ iff $\tilde{\mu}^{*}(X)=\tilde{\mu}(\omega X)$ and $X$ is $\tilde{\mu}^{*}$-measurable. (Since $X$ is normal, $\beta X=\omega X$.)

(2) If $X$ is 0 -dimensional and $T_{2}$, and if $\mu \in M_{R}(\mathbb{E})$, then $\mu \in M_{R}^{t}(\mathbb{E})$ iff $\tilde{\mu}^{*}(X)$ $=\tilde{\mu}\left(\beta_{0} X\right)$ and $X$ is $\tilde{\mu}^{*}$-measurable.

(3) If $X$ is $T_{1}$ and if $\mu \in M_{R}(\mathfrak{B})$, then $\mu \in M_{R}^{t}(\mathfrak{B})$ iff $\tilde{\mu}^{*}(X)=\tilde{\mu}\left(I_{R}(\mathfrak{B})\right)$ and $X$ is $\tilde{\mu}^{*}$-measurable.

(4) If $X$ is $T_{3 \frac{1}{2}}$ and if $\mu \in M_{R}(3)$, then $\mu \in M_{R}^{t}(3)$ iff $\tilde{\mu}^{*}(X)=\tilde{\mu}(\beta X)$ and $X$ is $\tilde{\mu}^{*}$-measurable.

We take pleasure in acknowledging our indebtedness to the referee for correcting a number of errors, for strengthening several results, and for vastly improving the entire presentation.

\section{References}

[1] A.D. Alexandroff, Additive set-functions in abstract spaces, Recueil Mathematique, T. 8 (50) \#2 (1940), pp. 307-342; T. 9 (51) \#3 (1941), pp. 563-621; T. 13 (55) (1943), pp. 139-268.

[2] G. Bachman and $\mathrm{Pa}$ o-Shing $\mathrm{Hsu}$, Extensions of lattice continuous maps to general Wallman spaces, Atti Naz. dei Lincei Rend. Cl. Sc. Fis. Mat. e Nat., Ser. VIII, Vol. LXII, fasc. 2, Feb. 1977, pp. 107-114.

[3] - and A: Sultan, Regular lattice measures: Mapping and spaces, Pacific J. Math. 67, \#2 (1976), pp. 291-321.

[4] - - Representations of linear functionals on spaces of continuous functions, repletions, and general measure extensions, J. Math. Anal. Appl. 67 (1979), pp. 277-293.

[5] - - On extensions of regular measures, Pacific J. Math. 86, \#2 (1980), pp. 389-395.

[6] B. Banaschewski, Uber nulldimensional Raume, Math. Nachr. 13 (1955), pp. 129-140.

[7] R. Engelking, Outline of General Topology, Amsterdam-New York 1968.

[8] R. J. Gardner, The regularity of Borel measure and Borel-measure compactness, Proc. London Math. Soc. 30 (1975), pp. 95-113.

[9] G. Gould and M. Mahowald, Measures on completely regular spaces, J. London Math. Soc. 37 (1962), pp. 103-111.

[10] P. Halmos, Measure Theory, Toronto-New York-London 1950

[11] J. Knowles, Measures on topological spaces, Proc. London Math. Soc. 17 (1967), pp. 139-156.

[12] W. Moran, Measures and mappings on topological spaces, Proc.London Math. Soc. 19 (1969), pp. 493-508.

[13] - The additivity of measures on completely regular spaces, J. London Math. Soc. 43 (1968), pp. 633-639.

[14] A. Sultan, Measure compactification and representations, Canad. J. Math. 30 (1) (1978), pp. $54-65$.

[15] M. Sze to, Measure repleteness and mapping preservations, J. Indian Math. Soc. (to appear)

[16] V.S. Varadarajan, Measures on topological spaces, Amer. Math. Soc. Trans. 48 (1965), pp. 161-228.

[17] H. Wallman, Lattices and topological spaces, Ann. of Math. 39 (1938), pp. 112-126.

POLYTECHNIC INSTITUTE OF NEW YORK

333 Jay Street Brooklyn, N. Y. 11201
COLLEGE OF STATEN ISLAND

THE CITY UNIVERSITY OF NEW YORK

130 Stuyvesant Place 10301

Received 16 March 1981;

in revised form 1 September 1982 